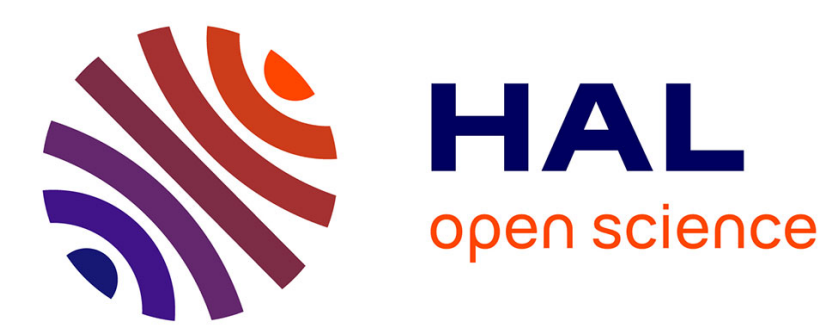

\title{
Some applications and methods of large deviations in finance and insurance \\ Huyen Pham
}

\section{To cite this version:}

Huyen Pham. Some applications and methods of large deviations in finance and insurance. 2007. hal-00131578v2

\author{
HAL Id: hal-00131578 \\ https://hal.science/hal-00131578v2
}

Preprint submitted on 19 Feb 2007

HAL is a multi-disciplinary open access archive for the deposit and dissemination of scientific research documents, whether they are published or not. The documents may come from teaching and research institutions in France or abroad, or from public or private research centers.
L'archive ouverte pluridisciplinaire HAL, est destinée au dépôt et à la diffusion de documents scientifiques de niveau recherche, publiés ou non, émanant des établissements d'enseignement et de recherche français ou étrangers, des laboratoires publics ou privés. 


\title{
Some applications and methods of large deviations in finance and insurance *
}

\author{
Huyên PHAM \\ Laboratoire de Probabilités et \\ Modèles Aléatoires \\ CNRS, UMR 7599 \\ Université Paris 7 \\ e-mail: pham@math.jussieu.fr \\ and Institut Universitaire de France
}

February 19, 2007

\begin{abstract}
In these notes, we present some methods and applications of large deviations to finance and insurance. We begin with the classical ruin problem related to the Cramer's theorem and give en extension to an insurance model with investment in stock market. We then describe how large deviation approximation and importance sampling are used in rare event simulation for option pricing. We finally focus on large deviations methods in risk management for the estimation of large portfolio losses in credit risk and portfolio performance in market investment.
\end{abstract}

Key words : large deviations, ruin problem, importance sampling, rare event simulation, exit probability, credit risk, portfolio performance.

MSC Classification (2000) : 60F10, 62P05, 65C05, 91B28, 91B30.

\footnotetext{
${ }^{*}$ Lectures from a Bachelier course at Institut Henri Poincaré, Paris, February 2007. I would like to thank the participants for their interest and comments.
} 


\section{Contents}

\begin{tabular}{|lll}
\hline 1 & Introduction & 3
\end{tabular}

2 Basic tools and results on large deviations 5

$2.1 \quad$ Laplace function and exponential change of measures . . . . . . . . . . . 5

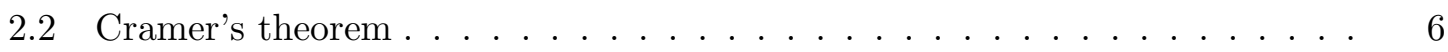

2.3 Some general principles in large deviations . . . . . . . . . . . . . . . . 10

\begin{tabular}{lll}
\hline & Ruin probabilities in risk theory & $\mathbf{1 5}$
\end{tabular}

3.1 The classical ruin problem . . . . . . . . . . . . . . . . . . . . . . . . . . . . . . . 15

3.1 .1 The insurance model . . . . . . . . . . . . . . . . . . . . . 15

3.1 .2 The Cramer-Lundberg estimate . . . . . . . . . . . . . . . . . . . . 16

$3.2 \quad$ Ruin probabilities and optimal investment . . . . . . . . . . . . . . . . . . . . . . . . . . . . . . . . 18

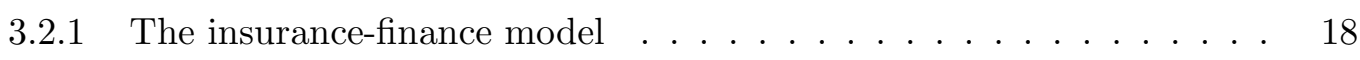

$3.2 .2 \quad$ Asymptotic ruin probability estimate . . . . . . . . . . . . . . . . 19

\begin{tabular}{|lll}
\hline & Large deviations and rare event simulation in option pricing & 23
\end{tabular}

$4.1 \quad$ Importance sampling and large deviations approximations . . . . . . . . . . 23

4.1 .1 Importance sampling for diffusions via Girsanov's theorem . . . . . . 23

4.1.2 Option pricing approximation with a Freidlin-Wentzell large devia-

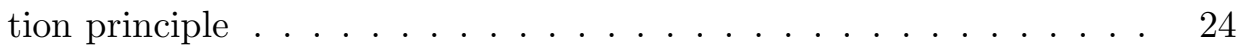

$4.1 .3 \quad$ Change of drift via Varadhan-Laplace principle . . . . . . . . . . . . 27

$4.2 \quad$ Computation of barrier crossing probabilities and sharp large deviations . . 30

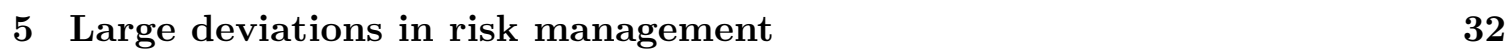

$5.1 \quad$ Large portfolio losses in credit risk $\ldots \ldots \ldots \ldots \ldots . \ldots \ldots$

$5.1 .1 \quad$ Portfolio credit risk in a single factor normal copula model . . . . . 32

5.1 .2 Independent obligors $\ldots \ldots \ldots \ldots . \ldots . \ldots . \ldots . \ldots 33$

5.1 .3 Dependent obligors . . . . . . . . . . . . . . . . . . . 34

$5.2 \quad$ A large deviations approach to optimal long term investment . . . . . . . . 39

$5.2 .1 \quad$ An asymptotic outperforming benchmark criterion . . . . . . . . . . 39

$5.2 .2 \quad$ Duality to the large deviations control problem . . . . . . . . . . . . 41

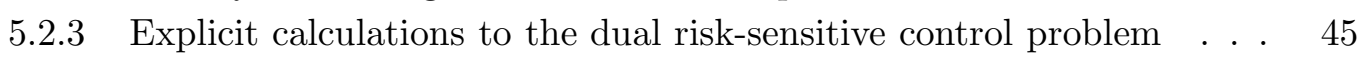

$\begin{array}{ll}6 & \text { Conclusion }\end{array} 49$ 


\section{Introduction}

The area of large deviations is a set of asymptotic results on rare event probabilities and a set of methods to derive such results. Large deviations is a very active area in applied probability, and questions related to extremal events both in finance and insurance applications, play an increasingly important role. For instance, recent applications of interest concern ruin probabilities in risk theory, risk management, option pricing, and simulation of quantities involved in this context.

Large deviations appear historically in insurance mathematics with the ruin probability estimation problem within the classical Cramer-Lundberg model. The problem was then subsequently extended to more general models involving for example Lévy risk processes. In finance, large deviations arise in various contexts. They occur in risk management for the computation of the probability of large losses of a portfolio subject to market risk or the default probabilities of a portfolio under credit risk. Large deviations methods are largely used in rare events simulation and so appear naturally in the approximation of option pricing, in particular for barrier options and far from the money options.

We illustrate our purpose with the following toy example. Let $X$ be a (real-valued) random variable, and consider the problem of computing or estimating $\mathbb{P}[X>\ell]$, the probability that $X$ exceeds some level $\ell$. In finance, we may think of $X$ as the loss of a portfolio subject to market or credit risk, and we are interested in the probability of large loss or default probability. The r.v. $X$ may also correspond to the terminal value of a stock price, and the quantity $\mathbb{P}[X>\ell]$ appears typically in the computation of a call or barrier option, with a small probability of payoff when the option is far from the money or the barrier $\ell$ is large. To estimate $p=\mathbb{P}[X>\ell]$, a basic technique is Monte Carlo simulation : generate $n$ independent copies $X_{1}, \ldots, X_{n}$ of $X$, and use the sample mean :

$$
\bar{S}_{n}=\frac{1}{n} \sum_{i=1}^{n} Y_{i}, \quad \text { with } Y_{i}=1_{X_{i}>\ell} .
$$

The convergence of this estimate (when $n \rightarrow \infty$ ) follows from the law of large numbers, while the standard rate of convergence is given, via the central limit theorem, in terms of the variance $v=p(1-p)$ of $Y_{i}$ :

$$
\mathbb{P}\left[\left|\bar{S}_{n}-p\right| \geq \frac{a}{\sqrt{n}}\right] \rightarrow 2 \Phi\left(-\frac{a}{\sqrt{v}}\right),
$$

where $\Phi$ is the cumulative distribution function of the standard normal law. Furthermore, the convergence of the estimator $\bar{S}_{n}$ is precised with the large deviation result, known here as the Cramer's theorem, which is concerned with approximation of rare event probabilities $\mathbb{P}\left[\bar{S}_{n} \in A\right]$, and typically states that

$$
\mathbb{P}\left[\left|\bar{S}_{n}-p\right| \geq a\right] \simeq C e^{-\gamma n},
$$

for some constants $C$ and $\gamma$.

Let us now turn again to the estimation of $p=\mathbb{P}[X>\ell]$. As mentioned above, the rate of convergence of the naive estimator $\bar{S}_{n}$ is determined by :

$$
\operatorname{Var}\left(\bar{S}_{n}\right)=\frac{\operatorname{Var}\left(1_{X>\ell}\right)}{n}=\frac{p(1-p)}{n},
$$


and the relative error is

$$
\text { relative error }=\frac{\text { standard deviation of } \bar{S}_{n}}{\text { mean of } \bar{S}_{n}}=\frac{\sqrt{p(1-p)}}{\sqrt{n} p} .
$$

Hence, if $p=\mathbb{P}[X>\ell]$ is small, and since $\sqrt{p-p^{2}} / p \rightarrow \infty$ as $p$ goes to zero, we see that a large sample size (i.e. $n$ ) is required for the estimator to achieve a reasonable relative error bound. This is a common occurence when estimating rare events. In order to improve the estimate of the tail probability $\mathbb{P}[X>\ell]$, one is tempted to use importance sampling to reduce variance, and hence speed up the computation by requiring fewer samples. This consists basically in changing measures to try to give more weight to "important" outcomes, (increase the default probability). Since large deviations theory also deals with rare events, we can see its strong link with importance sampling.

To make the idea concrete, consider again the problem of estimating $p=\mathbb{P}[X>\ell]$, and suppose that $X$ has distribution $\mu(d x)$. Let us look at an alternative sampling distribution $\nu(d x)$ absolutely continuous with respect to $\mu$, with density $f(x)=d \nu / d \mu(x)$. The tail probability can then be written as :

$$
p=\mathbb{P}[X>\ell]=\int 1_{x>\ell} \mu(d x)=\int 1_{x>\ell} \phi(x) \nu(d x)=\mathbb{E}_{\nu}\left[1_{X>\ell} \phi(X)\right],
$$

where $\phi=1 / f$, and $\mathbb{E}_{\nu}$ denotes the expectation under the measure $\nu$. By generating i.i.d. samples $\tilde{X}_{1}, \ldots, \tilde{X}_{n}, \ldots$ with distribution $\nu$, we have then an alternative unbiased and convergent estimate of $p$ with

$$
\tilde{S}_{n}=\frac{1}{n} \sum_{i=1}^{n} 1_{\tilde{X}_{i}>\ell} \phi\left(\tilde{X}_{i}\right),
$$

and whose rate of convergence is determined by

$$
\operatorname{Var}_{\nu}\left(\bar{S}_{n}\right)=\frac{1}{n} \int\left(1_{x>\ell}-p f(x)\right)^{2} \phi^{2}(x) \nu(d x) .
$$

The minimization of this quantity over all possible $\nu$ (or $f$ ) leads to a zero variance with the choice of a density $f(x)=1_{x>\ell} / p$. This is of course only a theoretical result since it requires the knowledge of $p$, the very thing we want to estimate! However, by noting that in this case $\nu(d x)=f(x) \mu(d x)=1_{x>\ell} \mu(d x) / \mathbb{P}[X>\ell]$ is nothing else than the conditional distribution of $X$ given $\{X>\ell\}$, this suggests to use an importance sampling change of measure that makes the rare event $\{X>\ell\}$ more likely. This method of suitable change of measure is also the key step in proving large deviation results.

The plan of these lectures is the following. In Section 2, we give some basic tools and results on large deviations, in particular the most classical result on large deviations, Cramer's theorem. We illustrate in Section 3 the first applications of large deviations to ruin problems in insurance industry, and give some extension to an insurance model with financial investment opportunity. Section 4 is concerned with the large deviations approximation for rare event simulation in option pricing, and we shall use asymptotic results from large deviations theory : Fredilin-Wentzell theory on sample path large deviations, and Varadhan's integral principle. Finally, Section 5 is devoted to applications of large deviations in risk management, where we use conditional and control variants of the Ellis-Gartner theorem. 


\section{Basic tools and results on large deviations}

\subsection{Laplace function and exponential change of measures}

If $X$ is a (real-valued) random variable on $(\Omega, \mathcal{F})$ with probability distribution $\mu(d x)$, the cumulant generating function (c.g.f.) of $\mu$ is the logarithm of the Laplace function of $X$, i.e. :

$$
\Gamma(\theta)=\ln \mathbb{E}\left[e^{\theta X}\right]=\ln \int e^{\theta x} \mu(d x) \in(-\infty, \infty], \quad \theta \in \mathbb{R} .
$$

Notice that $\Gamma(0)=0$, and $\Gamma$ is convex by Hölder inequality. We denote $\mathcal{D}(\Gamma)=\{\theta \in \mathbb{R}$ : $\Gamma(\theta)<\infty\}$, and for any $\theta \in \mathcal{D}(\Gamma)$, we define a probability measure $\mu_{\theta}$ on $\mathbb{R}$ by :

$$
\mu_{\theta}(d x)=\exp (\theta x-\Gamma(\theta)) \mu(d x) .
$$

Suppose that $X_{1}, \ldots, X_{n}, \ldots$, is an i.i.d. sequence of random variables with distribution $\mu$ and consider the new probability measure $\mathbb{P}_{\theta}$ on $(\Omega, \mathcal{F})$ with likelihood ratio evaluated at $\left(X_{1}, \ldots, X_{n}\right), n \in \mathbb{N}^{*}$, by :

$$
\frac{d \mathbb{P}_{\theta}}{d \mathbb{P}}\left(X_{1}, \ldots, X_{n}\right)=\prod_{i=1}^{n} \frac{d \mu_{\theta}}{d \mu}\left(X_{i}\right)=\exp \left(\theta \sum_{i=1}^{n} X_{i}-n \Gamma(\theta)\right) .
$$

By denoting $\mathbb{E}_{\theta}$ the corresponding expectation under $\mathbb{P}_{\theta}$, formula (2.2) means that for all $n \in \mathbb{N}^{*}$,

$$
\mathbb{E}\left[f\left(X_{1}, \ldots, X_{n}\right)\right]=\mathbb{E}_{\theta}\left[f\left(X_{1}, \ldots, X_{n}\right) \exp \left(-\theta \sum_{i=1}^{n} X_{i}+n \Gamma(\theta)\right)\right],
$$

for all Borel functions $f$ for which the expectation on the l.h.s. of (2.3) is finite. Moreover, the random variables $X_{1}, \ldots, X_{n}, n \in \mathbb{N}^{*}$, are i.i.d. with probability distribution $\mu_{\theta}$ under $\mathbb{P}_{\theta}$. Actually, the relation (2.3) extends from a fixed number of steps $n$ to a random number of steps, provided the random horizon is a stopping time. More precisely, if $\tau$ is a stopping time in $\mathbb{N}$ for $X_{1}, \ldots, X_{n}, \ldots$, i.e. the event $\{\tau<n\}$ is measurable with respect to the algebra generated by $\left\{X_{1}, \ldots, X_{n}\right\}$ for all $n$, then

$$
\mathbb{E}\left[f\left(X_{1}, \ldots, X_{\tau}\right) 1_{\tau<\infty}\right]=\mathbb{E}_{\theta}\left[f\left(X_{1}, \ldots, X_{\tau}\right) \exp \left(-\theta \sum_{i=1}^{\tau} X_{i}+\tau \Gamma(\theta)\right) 1_{\tau<\infty}\right]
$$

for all Borel functions $f$ for which the expectation on the l.h.s. of (2.4) is finite.

The cumulant generating function $\Gamma$ records some useful information on the probability distributions $\mu_{\theta}$. For example, $\Gamma^{\prime}(\theta)$ is the mean of $\mu_{\theta}$. Indeed, for any $\theta$ in the interior of $\mathcal{D}(\Gamma)$, differentiation yields by dominated convergence :

$$
\Gamma^{\prime}(\theta)=\frac{\mathbb{E}\left[X e^{\theta X}\right]}{\mathbb{E}\left[e^{\theta X}\right]}=\mathbb{E}[X \exp (\theta X-\Gamma(\theta))]=\mathbb{E}_{\theta}[X]
$$

A similar calculation shows that $\Gamma^{\prime \prime}(\theta)$ is the variance of $\mu_{\theta}$. Notice in particular that if 0 lies in the interior of $\mathcal{D}(\Gamma)$, then $\Gamma^{\prime}(0)=\mathbb{E}[X]$ and $\Gamma^{\prime \prime}(0)=\operatorname{Var}(X)$. 


\section{Bernoulli distribution}

Let $\mu$ the Bernoulli distribution of parameter $p$. Its c.g.f. is given by

$$
\Gamma(\theta)=\ln \left(1-p+p e^{\theta}\right) .
$$

A direct simple algebra calculation shows that $\mu_{\theta}$ is the Bernoulli distribution of parameter $p e^{\theta} /\left(1-p+p e^{\theta}\right)$.

\section{Poisson distribution}

Let $\mu$ the Poisson distribution of intensity $\lambda$. Its c.g.f. is given by

$$
\Gamma(\theta)=\lambda\left(e^{\theta}-1\right) .
$$

A direct simple algebra calculation shows that $\mu_{\theta}$ is the Poisson distribution of intensity $\lambda e^{\theta}$. Hence, the effect of the change of probability measure $\mathbb{P}_{\theta}$ is to multiply the intensity by a factor $e^{\theta}$.

\section{Normal distribution}

Let $\mu$ the normal distribution $\mathcal{N}\left(0, \sigma^{2}\right)$, whose c.g.f. is given by :

$$
\Gamma(\theta)=\frac{\theta^{2} \sigma^{2}}{2} .
$$

A direct simple algebra calculation shows that $\mu_{\theta}$ is the normal distribution $\mathcal{N}\left(\theta \sigma^{2}, \sigma^{2}\right)$. Hence, if $X_{1}, \ldots, X_{n}$ are i.i.d. with normal distribution $\mathcal{N}\left(0, \sigma^{2}\right)$, then under the change of measure $\mathbb{P}_{\theta}$ with likelihood ratio :

$$
\frac{d \mathbb{P}_{\theta}}{d \mathbb{P}}\left(X_{1}, \ldots, X_{n}\right)=\exp \left(\theta \sum_{i=1}^{n} X_{i}-n \frac{\theta^{2} \sigma^{2}}{2}\right),
$$

the random variables $X_{1}, \ldots, X_{n}$ are i.i.d. with normal distribution $\mathcal{N}\left(\theta \sigma^{2}, \sigma^{2}\right)$ : the effect of $\mathbb{P}_{\theta}$ is to change the mean of $X_{i}$ from 0 to $\theta \sigma^{2}$. This result can be interpreted as the finite-dimensional version of Girsanov's theorem.

\section{Exponential distribution}

Let $\mu$ the exponential distribution of intensity $\lambda$. Its c.g.f. is given by

$$
\Gamma(\theta)=\left\{\begin{array}{cc}
\ln \left(\frac{\lambda}{\lambda-\theta}\right), & \theta<\lambda \\
\infty, & \theta \geq \lambda
\end{array}\right.
$$

A direct simple algebra calculation shows that for $\theta<\lambda, \mu_{\theta}$ is the exponential distribution of intensity $\lambda-\theta$. Hence, the effect of the change of probability measure $\mathbb{P}_{\theta}$ is to shift the intensity from $\lambda$ to $\lambda-\theta$.

\subsection{Cramer's theorem}

The most classical result in large deviations area is Cramer's theorem. This concerns large deviations associated with the empirical mean of i.i.d. random variables valued in a finitedimensional space. We do not state the Cramer's theorem in whole generality. Our purpose 
is to put emphasis on the methods used to derive such result. For simplicity, we consider the case of real-valued i.i.d. random variables $X_{i}$ with (nondegenerate) probability distribution $\mu$ of finite mean $\mathbb{E} X_{1}=\int x \mu(d x)<\infty$, and we introduce the random walk $S_{n}=\sum_{i=1}^{n} X_{i}$. It is well-known by the law of large numbers that the empirical mean $S_{n} / n$ converges in probability to $\bar{x}=\mathbb{E} X_{1}$, i.e. $\lim _{n} \mathbb{P}\left[S_{n} / n \in(\bar{x}-\varepsilon, \bar{x}+\varepsilon)\right]=1$ for all $\varepsilon>0$. Notice also, by the central limit theorem that $\lim _{n} \mathbb{P}\left[S_{n} / n \in[\bar{x}, \bar{x}+\varepsilon)\right]=1 / 2$ for all $\varepsilon>0$. Large deviations results focus on asymptotics for probabilities of rare events, for example of the form $\mathbb{P}\left[\frac{S_{n}}{n} \geq x\right]$ for $x>\mathbb{E} X_{1}$, and state that

$$
\mathbb{P}\left[\frac{S_{n}}{n} \geq x\right] \simeq C e^{-\gamma x},
$$

for some constants $C$ and $\gamma$ to be precised later. The symbol $\simeq$ means that the ratio is one in the limit (here when $n$ goes to infinity). The rate of convergence is characterized by the Fenchel-Legendre transform of the c.g.f. $\Gamma$ of $X_{1}$ :

$$
\Gamma^{*}(x)=\sup _{\theta \in \mathbb{R}}[\theta x-\Gamma(\theta)] \in[0, \infty], \quad x \in \mathbb{R} .
$$

As supremum of affine functions, $\Gamma^{*}$ is convex. The sup in the definition of $\Gamma^{*}$ can be evaluated by differentiation : for $x \in \mathbb{R}$, if $\theta=\theta(x)$ is solution to the saddle-point equation, $x=\Gamma^{\prime}(\theta)$, then $\Gamma^{*}(x)=\theta x-\Gamma(\theta)$. Notice, from (2.5), that the exponential change of measure $\mathbb{P}_{\theta}$ put the expectation of $X_{1}$ to $x$. Actually, exponential change of measure is a key tool in large deviations methods. The idea is to select a measure under which the rare event is no longer rare, so that the rate of decrease of the original probability is given by the rate of decrease of the likelihood ratio. This particular change of measure is intended to approximate the most likely way for the rare event to occur.

By Jensen's inequality, we show that $\Gamma^{*}\left(\mathbb{E} X_{1}\right)=0$. This implies that for all $x \geq \mathbb{E} X_{1}$, $\Gamma^{*}(x)=\sup _{\theta \geq 0}[\theta x-\Gamma(\theta)]$, and so $\Gamma^{*}$ is nondecreasing on $\left[\mathbb{E} X_{1}, \infty\right)$.

Theorem 2.1 (Cramer's theorem)

For any $x \geq \mathbb{E} X_{1}$, we have

$$
\lim _{n \rightarrow \infty} \frac{1}{n} \ln \mathbb{P}\left[\frac{S_{n}}{n} \geq x\right]=-\Gamma^{*}(x)=-\inf _{y \geq x} \Gamma^{*}(y) .
$$

Proof. 1) Upper bound. The main step in the upper bound $\leq$ of (2.6) is based on Chebichev inequality combined with the i.i.d. assumption on the $X_{i}$ :

$$
\mathbb{P}\left[\frac{S_{n}}{n} \geq x\right]=\mathbb{E}\left[1_{\left.\frac{S_{n} \geq x}{n}\right]} \leq \mathbb{E}\left[e^{\theta\left(S_{n}-n x\right)}\right]=\exp (n \Gamma(\theta)-\theta n x), \quad \forall \theta \geq 0 .\right.
$$

By taking the infimum over $\theta \geq 0$, and since $\Gamma^{*}(x)=\sup _{\theta \geq 0}[\theta x-\Gamma(\theta)]$ for $x \geq \mathbb{E} X_{1}$, we then obtain

$$
\mathbb{P}\left[\frac{S_{n}}{n} \geq x\right] \leq \exp \left(-n \Gamma^{*}(x)\right) .
$$

and so in particular the upper bound $\leq$ of (2.6). 
2) Lower bound. Since $\mathbb{P}\left[\frac{S_{n}}{n} \geq x\right] \geq \mathbb{P}\left[\frac{S_{n}}{n} \in[x, x+\varepsilon)\right]$, for all $\varepsilon>0$, it suffices to show that

$$
\lim _{\varepsilon \rightarrow 0} \liminf _{n \rightarrow \infty} \frac{1}{n} \ln \mathbb{P}\left[\frac{S_{n}}{n} \in[x, x+\varepsilon)\right] \geq-\Gamma^{*}(x) .
$$

Suppose that $\mu$ is supported on a bounded support so that $\Gamma$ is finite everywhere. Suppose first that there exists a solution $\theta=\theta(x)>0$ to the saddle-point equation : $\Gamma^{\prime}(\theta)=x$, i.e. attaining the supremum in $\Gamma^{*}(x)=\theta(x) x-\Gamma(\theta(x))$. The key step is now to introduce the new probability distribution $\mu_{\theta}$ as in (2.1) and $\mathbb{P}_{\theta}$ the corresponding probability measure on $(\Omega, \mathcal{F})$ with likelihood ratio :

$$
\frac{d \mathbb{P}_{\theta}}{d \mathbb{P}}=\prod_{i=1}^{n} \frac{d \mu_{\theta}}{d \mu}\left(X_{i}\right)=\exp \left(\theta S_{n}-n \Gamma(\theta)\right) .
$$

Then, we have by (2.3) and for all $\varepsilon>0$ :

$$
\begin{aligned}
\mathbb{P}\left[\frac{S_{n}}{n} \in[x, x+\varepsilon)\right] & =\mathbb{E}_{\theta}\left[\exp \left(-\theta S_{n}+n \Gamma(\theta)\right) 1_{\frac{S_{n}}{n} \in[x, x+\varepsilon)}\right] \\
& =e^{-n(\theta x-\Gamma(\theta))} \mathbb{E}_{\theta}\left[\exp \left(-n \theta\left(\frac{S_{n}}{n}-x\right)\right) 1_{\frac{S_{n}}{n} \in[x, x+\varepsilon)}\right] \\
& \geq e^{-n(\theta x-\Gamma(\theta))} e^{-n|\theta| \varepsilon} \mathbb{P}_{\theta}\left[\frac{S_{n}}{n} \in[x, x+\varepsilon)\right]
\end{aligned}
$$

and so

$$
\frac{1}{n} \ln \mathbb{P}\left[\frac{S_{n}}{n} \in[x, x+\varepsilon)\right] \geq-[\theta x-\Gamma(\theta)]-|\theta| \varepsilon+\frac{1}{n} \ln \mathbb{P}_{\theta}\left[\frac{S_{n}}{n} \in[x, x+\varepsilon)\right] .
$$

Now, since $\Gamma^{\prime}(\theta)=x$, we have $\mathbb{E}_{\theta}\left[X_{1}\right]=x$, and by the law of large numbers and CLT : $\lim _{n} \mathbb{P}_{\theta}\left[\frac{S_{n}}{n} \in[x, x+\varepsilon)\right]=1 / 2(>0)$. We also have $\Gamma^{*}(x)=\theta x-\Gamma(\theta)$. Therefore, by sending $n$ to infinity and then $\varepsilon$ to zero in (2.8), we get (2.7).

Now, if the supremum in $\Gamma^{*}(x)$ is not attained, we can find a sequence $\left(\theta_{k}\right)_{k} \nearrow \infty$, such that $\theta_{k} x-\Gamma\left(\theta_{k}\right) \rightarrow \Gamma^{*}(x)$. Since $\mathbb{E}\left[e^{\theta_{k}\left(X_{1}-x\right)} 1_{X_{1}<x}\right] \rightarrow 0$, we then get

$$
\mathbb{E}\left[e^{\theta_{k}\left(X_{1}-x\right)} 1_{X_{1} \geq x}\right] \rightarrow e^{-\Gamma^{*}(x)},
$$

as $k$ goes to infinity. This is possible only if $\mathbb{P}\left[X_{1}>x\right]=0$ and $\mathbb{P}\left[X_{1}=x\right]=e^{-\Gamma^{*}(x)}$. By the i.i.d. assumption on the $X_{i}$, this implies $\mathbb{P}\left[S_{n} / n \geq x\right] \geq\left(\mathbb{P}\left[X_{1} \geq x\right]\right)^{n}=e^{-n \Gamma^{*}(x)}$, which proves $(2.7)$.

Suppose now that $\mu$ is of unbounded support, and fix $M$ large enough s.t. $\mu([-M, M])$ $>0$. By the preceding proof, the lower bound (2.7) holds with the law of $S_{n} / n$ conditional on $\left\{\left|X_{i}\right| \leq M, i=1, \ldots, n\right\}$, and with a c.g.f. equal to the c.g.f. of the conditional law of $X_{1}$ given $\left|X_{1}\right| \leq M$ :

$$
\begin{aligned}
& \lim _{\varepsilon \rightarrow 0} \liminf _{n \rightarrow \infty} \frac{1}{n} \ln \mathbb{P}\left[\frac{S_{n}}{n} \in[x, x+\varepsilon)|| X_{i} \mid \leq M, i=1, \ldots, n\right] \\
\geq & -\tilde{\Gamma}_{M}^{*}(x):=-\sup _{\theta \in \mathbb{R}}\left[\theta x-\tilde{\Gamma}_{M}(\theta)\right],
\end{aligned}
$$


with $\tilde{\Gamma}_{M}(\theta)=\ln \mathbb{E}\left[e^{\theta X_{1}}|| X_{1} \mid \leq M\right]=\Gamma_{M}(\theta)-\ln \mu([-M, M]), \Gamma_{M}(\theta)=\ln \mathbb{E}\left[e^{\theta X_{1}} 1_{\left|X_{1}\right| \leq M}\right]$. Now, by writing from Bayes formula that $\mathbb{P}\left[\frac{S_{n}}{n} \in[x, x+\varepsilon)\right]=\mathbb{P}\left[\frac{S_{n}}{n} \in[x, x+\varepsilon)|| X_{i} \mid \leq\right.$ $M, i=1, \ldots, n] \cdot(\mu([-M, M]))^{n}$, we get with (2.9)

$$
\begin{aligned}
& \lim _{\varepsilon \rightarrow 0} \liminf _{n \rightarrow \infty} \frac{1}{n} \ln \mathbb{P}\left[\frac{S_{n}}{n} \in[x, x+\varepsilon)\right] \\
\geq & \lim _{\varepsilon \rightarrow 0} \liminf _{n \rightarrow \infty} \frac{1}{n} \ln \mathbb{P}\left[\frac{S_{n}}{n} \in[x, x+\varepsilon)|| X_{i} \mid \leq M, i=1, \ldots, n\right]+\ln \mu([-M, M]) \\
\geq & -\Gamma_{M}^{*}(x):=-\sup _{\theta \in \mathbb{R}}\left[\theta x-\Gamma_{M}(\theta)\right] .
\end{aligned}
$$

The required result is obtained by sending $M$ to infinity. Notice also finally that $\inf _{y \geq x} \Gamma^{*}(y)$ $=\Gamma^{*}(x)$ since $\Gamma^{*}$ is nondecreasing on $\left[\mathbb{E} X_{1}, \infty\right)$.

\section{Examples}

1) Bernoulli distribution : for $X_{1} \sim \mathcal{B}(p)$, we have $\Gamma^{*}(x)=x \ln \left(\frac{x}{p}\right)+(1-x) \ln \left(\frac{1-x}{1-p}\right)$ for $x$ $\in[0,1]$ and $\infty$ otherwise.

2) Poisson distribution : for $X_{1} \sim \mathcal{P}(\lambda)$, we have $\Gamma^{*}(x)=x \ln \left(\frac{x}{\lambda}\right)+\lambda-x$ for $x \geq 0$ and $\infty$ otherwise.

3) Normal distribution : for $X_{1} \sim \mathcal{N}\left(0, \sigma^{2}\right)$, we have $\Gamma^{*}(x)=\frac{x^{2}}{2 \sigma^{2}}, x \in \mathbb{R}$.

2) Exponential distribution : for $X_{1} \sim \mathcal{E}(\lambda)$, we have $\Gamma^{*}(x)=\lambda x-1-\ln (\lambda x)$ for $x>0$ and $\Gamma^{*}(x)=\infty$ otherwise.

Remark 2.1 Cramer's theorem possesses a multivariate counterpart dealing with the large deviations of the empirical means of i.i.d. random vectors in $\mathbb{R}^{d}$.

Remark 2.2 (Relation with importance sampling)

Fix $n$ and let us consider the estimation of $p_{n}=\mathbb{P}\left[S_{n} / n \geq x\right]$. A standard estimator for $p_{n}$ is the average with $N$ independent copies of $X=1_{S_{n} / n \geq x}$. However, as shown in the introduction, for large $n, p_{n}$ is small, and the relative error of this estimator is large. By using an exponential change of measure $\mathbb{P}_{\theta}$ with likelihood ratio

$$
\frac{d \mathbb{P}_{\theta}}{d \mathbb{P}}=\exp \left(\theta S_{n}-n \Gamma(\theta)\right)
$$

so that

$$
p_{n}=\mathbb{E}_{\theta}\left[\exp \left(-\theta S_{n}+n \Gamma(\theta)\right) 1_{\frac{S_{n}}{n} \geq x}\right],
$$

we have an importance sampling (IS) (unbiased) estimator of $p_{n}$, by taking the average of independent replications of

$$
\exp \left(-\theta S_{n}+n \Gamma(\theta)\right) 1_{\frac{S_{n}}{n} \geq x} .
$$

The parameter $\theta$ is chosen in order to minimize the variance of this estimator, or equivalently its second moment :

$$
\begin{aligned}
M_{n}^{2}(\theta, x) & =\mathbb{E}_{\theta}\left[\exp \left(-2 \theta S_{n}+2 n \Gamma(\theta)\right) 1_{\frac{S_{n}}{n} \geq x}\right] \\
& \leq \exp (-2 n(\theta x-\Gamma(\theta)))
\end{aligned}
$$


By noting from Cauchy-Schwarz's inequality that $M_{n}^{2}(\theta, x) \geq p_{n}^{2}=\mathbb{P}\left[S_{n} / n \geq x\right] \simeq C e^{-2 n \Gamma^{*}(x)}$ as $n$ goes to infinity, from Cramer's theorem, we see that the fastest possible exponential rate of decay of $M_{n}^{2}(\theta, x)$ is twice the rate of the probability itself, i.e. $2 \Gamma^{*}(x)$. Hence, from (2.10), and with the choice of $\theta=\theta_{x}$ s.t. $\Gamma^{*}(x)=\theta_{x} x-\Gamma\left(\theta_{x}\right)$, we get an asymptotic optimal IS estimator in the sense that :

$$
\lim _{n \rightarrow \infty} \frac{1}{n} \ln M_{n}^{2}\left(\theta_{x}, x\right)=2 \lim _{n \rightarrow \infty} \frac{1}{n} \ln p_{n} .
$$

This parameter $\theta_{x}$ is such that $\mathbb{E}_{\theta_{x}}\left[S_{n} / n\right]=x$ so that the event $\left\{S_{n} / n \geq x\right\}$ is no more rare under $\mathbb{P}_{\theta_{x}}$, and is precisely the parameter used in the derivation of the large deviations result in Cramer's theorem.

\subsection{Some general principles in large deviations}

In this section, we give some general principles in large deviations theory. We refer to the classical references [13] or [15] for a detailed treatment on the subject.

We first give the formal definition of a large deviation principle (LDP). Consider a sequence $\left\{Z^{\varepsilon}\right\}_{\varepsilon}$ on $(\Omega, \mathcal{F}, \mathbb{P})$ valued in some topological space $\mathcal{X}$. The LDP characterizes the limiting behaviour as $\varepsilon \rightarrow 0$ of the family of probability measures $\left\{\mathbb{P}\left[Z^{\varepsilon} \in d x\right]\right\}_{\varepsilon}$ on $\mathcal{X}$ in terms of a rate function. A rate function $I$ is a lower semicontinuous function mapping $I: \mathcal{X} \rightarrow[0, \infty]$. It is a good rate function if the level sets $\{x \in \mathcal{X}: I(x) \leq M\}$ are compact for all $M<\infty$.

The sequence $\left\{Z^{\varepsilon}\right\}_{\varepsilon}$ satisfies a LDP on $\mathcal{X}$ with rate function $I$ (and speed $\varepsilon$ ) if :

(i) Upper bound: for any closed subset $F$ of $\mathcal{X}$

$$
\limsup _{\varepsilon \rightarrow 0} \varepsilon \ln \mathbb{P}\left[Z^{\varepsilon} \in F\right] \leq-\inf _{x \in F} I(x) .
$$

(ii) Lower bound: for any open subset $G$ of $\mathcal{X}$

$$
\liminf _{\varepsilon \rightarrow 0} \varepsilon \ln \mathbb{P}\left[Z^{\varepsilon} \in G\right] \geq-\inf _{x \in G} I(x) .
$$

If $F$ is a subset of $\mathcal{X}$ s.t. $\inf _{x \in F^{\circ}} I(x)=\inf _{x \in \bar{F}} I(x):=I_{F}$, then

$$
\lim _{\varepsilon \rightarrow 0} \varepsilon \ln \mathbb{P}\left[Z^{\varepsilon} \in F\right]=-I_{F},
$$

which formally means that $\mathbb{P}\left[Z^{\varepsilon} \in F\right] \simeq C e^{-I_{F} / \varepsilon}$ for some constant $C$. The classical Cramer's theorem considered the case of the empirical mean $Z^{\varepsilon}=S_{n} / n$ of i.i.d. random variables in $\mathbb{R}^{d}$, with $\varepsilon=1 / n$. Further main results in large deviations theory are the Gärtner-Ellis theorem, which is a version of Cramer's theorem where independence is weakened to the existence of

$$
\Gamma(\theta):=\lim _{\varepsilon \rightarrow 0} \varepsilon \ln \mathbb{E}\left[e^{\frac{\theta \cdot Z^{\varepsilon}}{\varepsilon}}\right], \quad \theta \in \mathbb{R}^{d} .
$$

LDP is then stated for the sequence $\left\{Z^{\varepsilon}\right\}_{\varepsilon}$ with a rate function equal to the FenchelLegendre transform of $\Gamma$ :

$$
\Gamma^{*}(x)=\sup _{\theta \in \mathbb{R}^{d}}[\theta \cdot x-\Gamma(\theta)], \quad x \in \mathbb{R}^{d} .
$$


Other results in large deviations theory include Sanov's theorem, which gives rare events asymptotics for empirical distributions. In many problems, the interest is in rare events that depend on random process, and the corresponding asymptotics probabilities, usually called sample path large deviations, were developed by Freidlin-Wentzell and DonskerVaradhan. For instance, the problem of diffusion exit from a domain is an important application of Freidlin-Wentzell theory, and occurs naturally in finance, see Section 4.1. We briefly summarize these results. Let $\varepsilon>0$ a (small) positive parameter and consider the stochastic differential equation in $\mathbb{R}^{d}$ on some interval $[0, T]$,

$$
d X_{s}^{\varepsilon}=b_{\varepsilon}\left(s, X_{s}^{\varepsilon}\right) d s+\sqrt{\varepsilon} \sigma\left(s, X_{s}^{\varepsilon}\right) d W_{s},
$$

and suppose that there exists a Lipschitz function $b$ on $[0, T] \times \mathbb{R}^{d}$ s.t.

$$
\lim _{\varepsilon \rightarrow 0} b_{\varepsilon}=b
$$

uniformly on compact sets. Given an open set $\Gamma$ of $[0, T] \times \mathbb{R}^{d}$, we consider the exit time from $\Gamma$,

$$
\tau_{t, x}^{\varepsilon}=\inf \left\{s \geq t: X_{s}^{\varepsilon, t, x} \notin \Gamma\right\},
$$

and the corresponding exit probability

$$
v_{\varepsilon}(t, x)=\mathbb{P}\left[\tau_{t, x}^{\varepsilon} \leq T\right], \quad(t, x) \in[0, T] \times \mathbb{R}^{d} .
$$

Here $X^{\varepsilon, t, x}$ denotes the solution to (2.11) starting from $x$ at time $t$. It is well-known that the process $X^{\varepsilon, t, x}$ converge to $X^{0, t, x}$ the solution to the ordinary differential equation

$$
d X_{s}^{0}=b\left(s, X_{s}^{0}\right) d s, \quad X_{t}^{0}=x .
$$

In order to ensure that $v_{\varepsilon}$ goes to zero, we assume that for all $t \in[0, T]$,

$$
\text { (H) } \quad x \in \Gamma \Longrightarrow X_{s}^{0, t, x} \in \Gamma, \quad \forall s \in[t, T] .
$$

Indeed, under $\mathbf{( H )}$, the system (2.11) tends, when $\varepsilon$ is small, to stay inside $\Gamma$, so that the event $\left\{\tau_{t, x}^{\varepsilon} \leq T\right\}$ is rare. The large deviations asymptotics of $v_{\varepsilon}(t, x)$, when $\varepsilon$ goes to zero, was initiated by Varadhan and Freidlin-Wentzell by probabilistic arguments. An alternative approach, introduced by Fleming, connects this theory with optimal control and Bellman equation, and is developed within the theory of viscosity solutions, see e.g. 9]. We sketch here this approach. It is well-known that the function $v_{\varepsilon}$ satisfies the linear PDE

$$
\frac{\partial v_{\varepsilon}}{\partial t}+b_{\varepsilon}(t, x) \cdot D_{x} v_{\varepsilon}+\frac{\varepsilon}{2} \operatorname{tr}\left(\sigma \sigma^{\prime}(t, x) D_{x}^{2} v_{\varepsilon}\right)=0, \quad(t, x) \in[0, T) \times \Gamma
$$

together with the boundary conditions

$$
\begin{aligned}
v_{\varepsilon}(t, x) & =1, \quad(t, x) \in[0, T) \times \partial \Gamma \\
v_{\varepsilon}(T, x) & =0, \quad x \in \Gamma .
\end{aligned}
$$


Here $\partial \Gamma$ is the boundary of $\Gamma$. We now make the logarithm transformation

$$
V_{\varepsilon}=-\varepsilon \ln v_{\varepsilon}
$$

Then, after some straightforward derivation, (2.12) becomes the nonlinear PDE

$$
\begin{aligned}
-\frac{\partial V_{\varepsilon}}{\partial t}-b_{\varepsilon}(t, x) \cdot D_{x} & V_{\varepsilon}-\frac{\varepsilon}{2} \operatorname{tr}\left(\sigma \sigma^{\prime}(t, x) D_{x}^{2} V_{\varepsilon}\right) \\
+ & \frac{1}{2}\left(D_{x} V_{\varepsilon}\right)^{\prime} \sigma \sigma^{\prime}(t, x) D_{x} V_{\varepsilon}=0, \quad(t, x) \in[0, T) \times \Gamma,
\end{aligned}
$$

and the boundary data (2.13)-(2.14) become

$$
\begin{aligned}
V_{\varepsilon}(t, x) & =0, \quad(t, x) \in[0, T) \times \partial \Gamma \\
V_{\varepsilon}(T, x) & =\infty, \quad x \in \Gamma .
\end{aligned}
$$

At the limit $\varepsilon=0$, the PDE (2.15) becomes a first-order PDE

$$
-\frac{\partial V_{0}}{\partial t}-b(t, x) \cdot D_{x} V_{0}+\frac{1}{2}\left(D_{x} V_{0}\right)^{\prime} \sigma \sigma^{\prime}(t, x) D_{x} V_{0}=0, \quad(t, x) \in[0, T) \times \Gamma,
$$

with the boundary data (2.16)-(2.17). By PDE-viscosity solutions methods and comparison results, we can prove (see e.g. [9] or [19]) that $V_{\varepsilon}$ converges uniformly on compact subsets of $[0, T) \times \Gamma$, as $\varepsilon$ goes to zero, to $V_{0}$ the unique viscosity solution to (2.18) with the boundary data (2.16)-(2.17). Moreover, $V_{0}$ has a representation in terms of control problem. Consider the Hamiltonian function

$$
H(t, x, p)=-b(t, x) \cdot p+\frac{1}{2} p^{\prime} \sigma \sigma^{\prime}(t, x) p, \quad(t, x, p) \in[0, T] \times \Gamma \times \mathbb{R}^{d},
$$

which is quadratic and in particular convex in $p$. Then, using the Legendre transform, we may rewrite

$$
H(t, x, p)=\sup _{q \in \mathbb{R}^{d}}\left[-q \cdot p-H^{*}(t, x, q)\right]
$$

where

$$
\begin{aligned}
H^{*}(t, x, q) & =\sup _{p \in \mathbb{R}^{d}}[-p \cdot q-H(t, x, p)] \\
& =\frac{1}{2}(q-b(t, x))^{\prime}\left(\sigma \sigma^{\prime}(t, x)\right)^{-1}(q-b(t, x)), \quad(t, x, q) \in[0, T] \times \Gamma \times \mathbb{R}^{d} .
\end{aligned}
$$

Hence, the PDE (2.18) is rewritten as

$$
\frac{\partial V_{0}}{\partial t}+\inf _{q \in \mathbb{R}^{d}}\left[q \cdot D_{x} V_{0}+H^{*}(t, x, q)\right]=0, \quad(t, x) \in[0, T) \times \Gamma,
$$

which, together with the boundary data (2.16)-(2.17), is associated to the value function for the following calculus of variations problem : for an absolutely continuous function $x($. 
on $[0, T)$ and valued in $\mathbb{R}^{d}$, i.e. $x \in H_{l o c}^{1}\left([0, T], \mathbb{R}^{d}\right)$, we denote $\dot{x}(u)=q_{u}$ its time derivative, and $\tau(x)$ the exit time of $x($.$) from \Gamma$. Then,

$$
\begin{aligned}
V_{0}(t, x) & =\inf _{x(.) \in \mathcal{A}(t, x)} \int_{t}^{T} H^{*}(u, x(u), \dot{x}(u)) d u, \quad(t, x) \in[0, T) \times \Gamma, \\
& =\inf _{x(.) \in \mathcal{A}(t, x)} \int_{t}^{T} \frac{1}{2}(\dot{x}(u)-b(u, x(u)))^{\prime}\left(\sigma \sigma^{\prime}(u, x(u))\right)^{-1}(\dot{x}(u)-b(u, x(u))) d u
\end{aligned}
$$

where

$$
\mathcal{A}(t, x)=\left\{x(.) \in H_{l o c}^{1}\left([0, T], \mathbb{R}^{d}\right): x(t)=x \text { and } \tau(x) \leq T\right\} .
$$

The large deviations result is then stated as

$$
\lim _{\varepsilon \rightarrow 0} \varepsilon \ln v_{\varepsilon}(t, x)=-V_{0}(t, x)
$$

and the above limit holds uniformly on compact subsets of $[0, T) \times \Gamma$. A more precise result may be obtained, which allows to remove the above log estimate. This type of result is developed in [17, and is called sharp large deviations estimate. It states asymptotic expansion (in $\varepsilon$ ) of the exit probability for points $(t, x)$ belonging to a set $N$ of $\left[0, T^{\prime}\right] \times \Gamma$ for some $T^{\prime}<T$, open in the relative topology, and s.t. $V_{0} \in C^{\infty}(N)$. Then, under the condition that

$$
b_{\varepsilon}=b+\varepsilon b_{1}+0\left(\varepsilon^{2}\right)
$$

one has

$$
v_{\varepsilon}(t, x)=\exp \left(-\frac{V_{0}(t, x)}{\varepsilon}-w(t, x)\right)(1+O(\varepsilon)),
$$

uniformly on compact sets of $N$, where $w$ is solution to the PDE problem

$$
\begin{aligned}
-\frac{\partial W}{\partial t}-\left(b-\sigma \sigma^{\prime} D_{x} V_{0}\right) \cdot D_{x} w & =\frac{1}{2} \operatorname{tr}\left(\sigma \sigma^{\prime} D_{x}^{2} V_{0}\right)+b_{1} \cdot D_{x} V_{0} \quad \text { in } N \\
w(t, x) & =0 \text { on }([0, T) \times \partial \Gamma) \cup \bar{N} .
\end{aligned}
$$

The function $w$ may be represented as

$$
w(t, x)=\int_{t}^{\rho}\left(\frac{1}{2} \operatorname{tr}\left(\sigma \sigma^{\prime} D_{x}^{2} V_{0}\right)+b_{1} \cdot D_{x} V_{0}\right)(s, \xi(s)) d s,
$$

where $\xi$ is the solution to

$$
\dot{\xi}(s)=\left(b-\sigma \sigma^{\prime} D_{x} V_{0}\right)(s, \xi(s)), \quad \xi(t)=x,
$$

and $\rho$ is the exit time (after $t)$ of $(s, \xi(s))$ from $N$.

We shall develop more in detail in the next sections some applications of the GärtnerEllis and Freidlin-Wentzell theories in finance.

We end this paragraph by stating the important Varadhan's integral formula, which involves the asymptotics behavior of certain expectations. It extends the well-known method 
of Laplace for studying the asymptotics of certain integrals on $\mathbb{R}$ : given a continuous function $\varphi$ from $[0,1]$ into $\mathbb{R}$, Laplace's method states that

$$
\lim _{n \rightarrow \infty} \frac{1}{n} \ln \int_{0}^{1} e^{n \varphi(x)} d x=\max _{x \in[0,1]} \varphi(x) .
$$

Varadhan result's is formulated as follows :

Theorem 2.2 (Varadhan) Suppose that $\left\{Z^{\varepsilon}\right\}_{\varepsilon}$ satisfies a LDP on $\mathcal{X}$ with good rate function $I$, and let $\varphi: \mathcal{X} \rightarrow \mathbb{R}$ be any continuous function s.t. the following moment condition holds for some $\gamma>1$ :

$$
\limsup _{\varepsilon \rightarrow 0} \varepsilon \ln \mathbb{E}\left[e^{\gamma \varphi\left(Z^{\varepsilon}\right) / \varepsilon}\right]<\infty
$$

Then,

$$
\lim _{\varepsilon \rightarrow 0} \varepsilon \ln \mathbb{E}\left[e^{\varphi\left(Z^{\varepsilon}\right) / \varepsilon}\right]=\sup _{x \in \mathcal{X}}[\varphi(x)-I(x)] .
$$

Proof. (a) For simplicity, we show the inequality $\leq$ in $(2.20)$ when $\varphi$ is bounded on $\mathcal{X}$. Hence, there exists $M \in(0, \infty)$ s.t. $-M \leq \varphi(x) \leq M$ for all $x \in \mathcal{X}$. For $N$ positive integer, and $j \in\{1, \ldots, N\}$, we consider the closed subsets of $\mathcal{X}$

$$
F_{N, j}=\left\{x \in \mathcal{X}:-M+\frac{2(j-1) M}{N} \leq \varphi(x) \leq-M+\frac{2 j M}{N}\right\},
$$

so that $\cup_{j=1}^{N} F_{N, j}=\mathcal{X}$. We then have from the large deviations upper bound on $\left(Z^{\varepsilon}\right)$,

$$
\begin{aligned}
\limsup _{\varepsilon \rightarrow 0} \varepsilon \ln \mathbb{E}\left[e^{\varphi\left(Z^{\varepsilon}\right) / \varepsilon}\right] & =\limsup _{\varepsilon \rightarrow 0} \varepsilon \ln \int_{\mathcal{X}} e^{\varphi\left(Z^{\varepsilon}\right) / \varepsilon} \mathbb{P}\left[Z^{\varepsilon} \in d x\right] \\
& \leq \limsup _{\varepsilon \rightarrow 0} \varepsilon \ln \left(\sum_{j=1}^{N} \int_{F_{N, j}} e^{\varphi\left(Z^{\varepsilon}\right) / \varepsilon} \mathbb{P}\left[Z^{\varepsilon} \in d x\right]\right) \\
& \leq \limsup _{\varepsilon \rightarrow 0} \varepsilon \ln \left(\sum_{j=1}^{N} e^{(-M+2 j M / N) / \varepsilon} \mathbb{P}\left[Z^{\varepsilon} \in F_{N, j}\right]\right) \\
& \leq \limsup _{\varepsilon \rightarrow 0} \varepsilon \ln \left(\max _{j=1, \ldots, N} e^{(-M+2 j M / N) / \varepsilon} \mathbb{P}\left[Z^{\varepsilon} \in F_{N, j}\right]\right) \\
& \leq \max _{j=1, \ldots, N}\left(-M+\frac{2 j M}{N}+\limsup _{\varepsilon \rightarrow 0} \varepsilon \ln \mathbb{P}\left[Z^{\varepsilon} \in F_{N, j}\right]\right) \\
& \leq \max _{j=1, \ldots, N}\left(-M+\frac{2 j M}{N}+\sup _{x \in F_{N, j}}[-I(x)]\right) \\
& \leq \max _{j=1, \ldots, N}\left(-M+\frac{2 j M}{N}+\sup _{x \in F_{N, j}}[\varphi(x)-I(x)]-\inf _{x \in F_{N, j}} \varphi(x)\right) \\
& \leq \sup _{x \in F_{N, j}}[\varphi(x)-I(x)]+\frac{2 M}{N} .
\end{aligned}
$$

By sending $N$ to infinity, we get the inequality $\leq$ in $(2.20)$. 
(b) To prove the reverse inequality, we fix an arbitrary point $x_{0} \in \mathcal{X}$, an arbitrary $\delta>0$, and we consider the open set $G=\left\{x \in \mathcal{X}: \varphi(x)>\varphi\left(x_{0}\right)-\delta\right\}$. Then, we have from the large deviations lower bound on $\left(Z^{\varepsilon}\right)$,

$$
\begin{aligned}
\liminf _{\varepsilon \rightarrow 0} \varepsilon \ln \mathbb{E}\left[e^{\varphi\left(Z^{\varepsilon}\right) / \varepsilon}\right] & \geq \liminf _{\varepsilon \rightarrow 0} \varepsilon \ln \mathbb{E}\left[e^{\varphi\left(Z^{\varepsilon}\right) / \varepsilon} 1_{\left.Z^{\varepsilon} \in G\right]}\right. \\
& \geq \varphi\left(x_{0}\right)-\delta+\liminf _{\varepsilon \rightarrow 0} \varepsilon \ln \mathbb{P}\left[Z^{\varepsilon} \in G\right] \\
& \geq \varphi\left(x_{0}\right)-\delta-\inf _{x \in G} I(x) \\
& \geq \varphi\left(x_{0}\right)-I\left(x_{0}\right)-\delta .
\end{aligned}
$$

Since $x_{0} \in \mathcal{X}$ and $\delta>0$ are arbitrary, we get the required result.

Remark 2.3 The relation (2.20) has the following interpretation. By writing formally the LDP for $\left(Z^{\varepsilon}\right)$ with rate function $I$ as $\mathbb{P}\left[Z^{\varepsilon} \in d x\right] \simeq e^{-I(x) / \varepsilon} d x$, we can write

$$
\begin{aligned}
\mathbb{E}\left[e^{\varphi\left(Z^{\varepsilon}\right) / \varepsilon}\right]=\int e^{\varphi(x) / \varepsilon} \mathbb{P}\left[Z^{\varepsilon} \in d x\right] & \simeq \int e^{(\varphi(x)-I(x)) / \varepsilon} d x \\
& \simeq C \exp \left(\frac{\sup _{x \in \mathcal{X}}(\varphi(x)-I(x))}{\varepsilon}\right) .
\end{aligned}
$$

As in Laplace's method, Varadhan's formula states that to exponential order, the main contribution to the integral is due to the largest value of the exponent.

\section{Ruin probabilities in risk theory}

\subsection{The classical ruin problem}

\subsubsection{The insurance model}

We consider an insurance company earning premiums at a constant rate $p$ per unit of time, and paying claims that arrive at the jumps of a Poisson process with intensity $\lambda$. We denote by $N_{t}$ the number of claims arriving in $[0, t]$, by $T_{n}, n \geq 1$, the arrival times of the claim, and by $\xi_{1}=T_{1}, \xi_{n}=T_{n}-T_{n-1}, n \geq 2$, the interarrival times, which are then i.i.d. exponentially distributed with finite mean $E \xi_{1}=1 / \lambda$. The size of the $n$-th claim is denoted $Y_{n}$, and we assume that the claim sizes $Y_{n}, n \in \mathbb{N}^{*}$, are (positive) i.i.d., and independent of the Poisson process. Starting from an initial reserve $x>0$, the risk reserve process $X_{t}$ $=X_{t}^{x}, t \geq 0$, of the insurance company is then given by :

$$
X_{t}^{x}=x+p t-\sum_{i=1}^{N_{t}} Y_{i} .
$$

The probability of ruin with infinite horizon is

$$
\psi(x)=\mathbb{P}\left[\tau_{x}<\infty\right]
$$

where $\tau_{x}=\inf \left\{t \geq 0: X_{t}^{x}<0\right\}$ is the time of ruin. We are interested in the estimation of the ruin probability, in particular for large values of the initial reserve. 


\subsubsection{The Cramer-Lundberg estimate}

The Cramer-Lundberg approximation concerns the estimation of the ruin probability $\psi(x)$, and is one of the most celebrated result of risk theory. There are several approaches for deriving such a result. We follow in this paragraph a method based on large deviations arguments and change of probability measures.

First, we easily see, by the strong law of large numbers, that

$$
\frac{1}{t} \sum_{i=1}^{N_{t}} Y_{i} \rightarrow \rho \text { a.s. }, \quad t \rightarrow \infty,
$$

where $\rho=\lambda \mathbb{E}\left[Y_{1}\right]>0$ is interpreted as the average amount of claim per unit of time. The safety loading $\eta$ plays a key role in ruin probability. It is defined as the relative amount by which the premium rate exceeds $\rho$ :

$$
\eta=\frac{p-\rho}{\rho} \Longleftrightarrow p=(1+\eta) \rho
$$

Hence $X_{t}^{x} / t \rightarrow p-\rho=\rho \eta$ when $t$ goes to infinity. Therefore, if $\eta<0, X_{t}^{x} \rightarrow-\infty$, and we clearly have $\psi(x)=1$ for all $x$. For $\eta=0$, we can also show that $\lim \sup X_{t}^{x}=-\infty$ so that $\psi(x)=1$. In the sequel, we make the net profit assumption :

$$
\eta=\frac{p-\lambda \mathbb{E}\left[Y_{1}\right]}{\lambda \mathbb{E}\left[Y_{1}\right]}>0
$$

which ensures that the probability of ruin is less than 1.

Since ruin may occur only at the arrival of a claim, i.e. when $X$ jumps downwards, it suffices to consider the discrete-time process embedded at the jumps of the Poisson process. We then define the discrete-time process $X_{T_{n}}^{x}, n \geq 1$, so that

$$
\psi(x)=\mathbb{P}\left[\sigma_{x}<\infty\right],
$$

where $\sigma_{x}=\inf \left\{n \geq 1: X_{T_{n}}^{x}<0\right\}=\inf \left\{n \geq 1: S_{n}>x\right\}$, and $S_{n}=x-X_{T_{n}}^{x}$ is the net payout up to the $n$-th claim and given by the random walk :

$$
S_{n}=Z_{1}+\ldots+Z_{n}, \quad Z_{i}=Y_{i}-p \xi_{i}, \quad i \in \mathbb{N}^{*} .
$$

The r.v. $Z_{i}$ are i.i.d. and satisfy under the net profit condition, $\mathbb{E}\left[Z_{1}\right]<0$. We denote by $\Gamma_{Z}$ the c.g.f. of the $Z_{i}$, and we see that by independence of $Y_{i}$ and $\xi_{i}$ :

$$
\begin{aligned}
\Gamma_{Z}(\theta) & =\Gamma_{Y}(\theta)+\Gamma_{\xi}(-p \theta) \\
& =\Gamma_{Y}(\theta)+\ln \left(\frac{\lambda}{\lambda+p \theta}\right), \quad \theta>-\frac{\lambda}{p},
\end{aligned}
$$

where $\Gamma_{Y}$ (resp. $\left.\Gamma_{\xi}\right)$ is the c.g.f. of the $Y_{i}\left(\operatorname{resp} . \xi_{i}\right)$. For any $\theta$ in the domain of $\Gamma_{Z}$, we consider the exponential change of measure with parameter $\theta$, and since $\sigma_{x}$ is a stopping time in the filtration of $\left(Z_{1}, \ldots, Z_{n}\right)$, we apply formula (2.4) to write the ruin probability as an $\mathbb{E}_{\theta}$ expectation :

$$
\begin{aligned}
\psi(x) & =\mathbb{P}\left[\sigma_{x}<\infty\right]=\mathbb{E}\left[1_{\sigma_{x}<\infty}\right] \\
& =\mathbb{E}_{\theta}\left[1_{\sigma_{x}<\infty} \exp \left(-\theta S_{\sigma_{x}}+\sigma_{x} \Gamma_{Z}(\theta)\right)\right] .
\end{aligned}
$$


We now assume $Y$ has a light-tailed distribution, i.e. : there exists $\bar{\theta} \in(0, \infty]$ s.t. $\Gamma_{y}(\theta)<$ $\infty$ for $\theta<\bar{\theta}$, and $\Gamma_{Y}(\theta) \rightarrow \infty$ as $\theta \nearrow \bar{\theta}$. In this case, the c.g.f. $\Gamma_{Z}$ of the $Z_{i}$ is finite on $(-\lambda / p, \bar{\theta})$, it is differentiable in 0 with $\Gamma_{Z}^{\prime}(0)=\mathbb{E}\left[Z_{1}\right]<0$ under the net profit condition (3.2). Moreover, since $\mathbb{E}\left[Y_{1}\right]>0$ and $Y_{1}$ is independent of $\xi_{1}$, we see that $\mathbb{P}\left[Z_{1}>0\right]>0$, which implies that $\Gamma_{Z}(\theta)$ goes to infinity as $\theta$ goes to $\bar{\theta}$. By convexity of $\Gamma_{Z}$ and recalling that $\Gamma_{Z}(0)=0$, we deduce the existence of an unique $\theta_{L}>0$ s.t. $\Gamma_{Z}\left(\theta_{L}\right)=0$. This unique positive $\theta_{L}$ is the solution to the so-called Cramer-Lundberg equation :

$$
\Gamma_{Y}\left(\theta_{L}\right)+\ln \left(\frac{\lambda}{\lambda+p \theta_{L}}\right)=0
$$

which is also written equivalently in :

$$
\gamma_{Y}\left(\theta_{L}\right)=\frac{p \theta_{L}}{\lambda}
$$

where $\gamma_{Y}=\exp \left(\Gamma_{Y}(\theta)\right)-1=\int e^{\theta y} F_{Y}(d y)-1$ is the shifted $\left(\gamma_{Y}(0)=0\right)$ moment generating function of $Y_{i}$, and $F_{Y}$ is the distribution function of the claim sizes $Y_{i}$. $\theta_{L}$ is called adjustment coefficient (or sometimes Lundberg exponent). Notice also that by convexity of $\Gamma_{Z}$, we have $\Gamma_{Z}^{\prime}\left(\theta_{L}\right)>0$. Hence, under $\mathbb{P}_{\theta_{L}}$, the random walk has positive drift $\mathbb{E}_{\theta_{L}}\left[Z_{n}\right]=$ $\Gamma_{Z}^{\prime}\left(\theta_{L}\right)>0$, and this implies $\mathbb{P}_{\theta_{L}}\left[\sigma_{x}<\infty\right]=1$. For this choice of $\theta=\theta_{L}$, (3.3) becomes

$$
\psi(x)=\mathbb{E}_{\theta_{L}}\left[e^{-\theta_{L} S_{\sigma_{x}}}\right]=e^{-\theta_{L} x} \mathbb{E}_{\theta_{L}}\left[e^{-\theta_{L}\left(S_{\sigma_{x}}-x\right)}\right] .
$$

By noting that the overshoot $S_{\sigma_{x}}-x$ is nonnegative, we obtain the Lundberg's inequality on the ruin probability :

$$
\psi(x) \leq e^{-\theta_{L} x}, \quad \forall x>0 .
$$

Moreover, by renewal's theory, the overshoot $R^{x}=S_{\sigma_{x}}-x$ has a limit $R^{\infty}$ (in the sense of weak convergence with respect to $\mathbb{P}_{\theta_{L}}$ ), when $x$ goes to infinity, and therefore $\mathbb{E}_{\theta_{L}}\left[e^{-\theta_{L} R^{x}}\right]$ converges to some positive constant $C$. We then get the classical approximation for large values of the initial reserve :

$$
\psi(x) \simeq C e^{-\theta_{L} x},
$$

as $x \rightarrow \infty$, which implies a large deviation type estimation

$$
\lim _{x \rightarrow \infty} \frac{1}{x} \ln \psi(x)=-\theta_{L} .
$$

Further details and extensions can be found in [3] or [16]. They concern more general processes (e.g. Levy proceses) for the risk reserve, heavy-tailed distribution for the claim size ... In the next paragraph, we study an extension of the classical ruin problem, developed by [23] and [31], where the insurer has the additional opportunity to invest in a stock market.

\section{Application : Importance sampling for the ruin probability estimation}

From the perspective of estimation of the ruin probability $\psi(x)$, and by choosing the Lundberg exponent $\theta_{L}$, we have an unbiased estimator with the associated importance sampling estimator based on Monte-Carlo simulations of

$$
\psi(x)=\mathbb{E}_{\theta_{L}}\left[e^{-\theta_{L} S_{\sigma_{x}}}\right] .
$$


Since, obviously, $S_{\sigma_{x}}>x$, the second order moment of this estimator satisfies

$$
M^{2}\left(\theta_{L}, x\right)=\mathbb{E}_{\theta_{L}}\left[e^{-2 \theta_{L} S_{\sigma_{x}}}\right] \leq e^{-2 \theta_{L} x} .
$$

On the other hand, by Cauchy-Schwarz's inequality, the second moment of any unbiased estimator must be as large as the square of the ruin probability, and we have seen that this probability is $O\left(e^{-\theta_{L} x}\right)$. Therefore, the IS estimator based on $\theta_{L}$ is asymptotically optimal as $x \rightarrow \infty$ :

$$
\lim _{x \rightarrow \infty} \frac{1}{x} \ln M^{2}\left(\theta_{L}, x\right)=2 \lim _{x \rightarrow \infty} \frac{1}{x} \ln \psi(x)\left(=2 \theta_{L}\right) .
$$

Remark 3.1 For any $x>0, \theta>0$, consider the process

$$
M_{t}(x, \theta)=\exp \left(-\theta X_{t}^{x}\right), \quad t \geq 0 .
$$

where $X_{t}^{x}$ is the risk reserve process defined in (3.1). A standard calculation shows that for all $t \geq 0$,

$$
\mathbb{E}\left[M_{t}(0, \theta)\right]=e^{\left(\lambda \gamma_{Y}(\theta)-p \theta\right) t}
$$

Moreover, since $X_{t}^{x}$ has stationary independent increments, and by denoting $\mathbb{F}=\left(\mathcal{F}_{t}\right)_{t \geq 0}$ the filtration generated by the risk reserve $X$, we have for all $0 \leq t \leq T$

$$
\begin{aligned}
\mathbb{E}\left[M_{T}(x, \theta) \mid \mathcal{F}_{t}\right] & =\mathbb{E}\left[e^{-\theta X_{T}^{x}} \mid \mathcal{F}_{t}\right] \\
& =M_{t}(x, \theta) \mathbb{E}\left[e^{-\theta\left(X_{T}^{x}-X_{t}^{x}\right)} \mid \mathcal{F}_{t}\right] \\
& =M_{t}(x, \theta) \mathbb{E}\left[M_{T-t}(0, \theta)\right] \\
& =M_{t}(x, \theta) e^{\left(\lambda \gamma_{Y}(\theta)-p \theta\right)(T-t)} .
\end{aligned}
$$

Hence, for the choice of $\theta=\theta_{L}$ : the adjustment coefficient, the process $M_{t}\left(x, \theta_{L}\right), t \geq 0$, is a $(\mathbb{P}, \mathbb{F})$-martingale. The use of this martingale property in the derivation of ruin estimate was initiated by Gerber [24]. We show in the next paragraph how to extend this idea for a ruin problem with investment in a stock market.

\subsection{Ruin probabilities and optimal investment}

\subsubsection{The insurance-finance model}

In the setting of the classical model described in the previous paragraph, we consider the additional feature that the insurance company is also allowed to invest in some stock market, modeled by a geometric brownian motion :

$$
d S_{t}=b S_{t} d t+\sigma S_{t} d W_{t}
$$

where $b, \sigma$ are constants, $\sigma>0$, and $W$ is a standard brownian motion, independent of the risk reserve $X$ as defined in (3.1). We denote by $\mathbb{F}=\left(\mathcal{F}_{t}\right)_{t \geq 0}$ the filtration generated by $X$ and $S$. The insurer may invest at any time $t$ an amount of money $\alpha_{t}$ in the stock, and the rest in the bond (which in the present model yields no interest). The set $\mathcal{A}$ of admissible 
investment strategies is defined as the set of $\mathbb{F}$-adapted processes $\alpha=\left(\alpha_{t}\right)$ s.t. $\int_{0}^{t} \alpha_{s}^{2} d s<$ $\infty$ a.s. Given an initial capital $x \geq 0$, and an admissible investment control $\alpha$, the insurer's wealth process can then be written as

$$
\begin{aligned}
V_{t}^{x, \alpha} & =X_{t}^{x}+\int_{0}^{t} \frac{\alpha_{u}}{S_{u}} d S_{u} \\
& =x+p t-\sum_{i=1}^{N_{t}} Y_{i}+\int_{0}^{t} \alpha_{u}\left(b d u+\sigma d W_{u}\right), \quad t \geq 0 .
\end{aligned}
$$

We define the infinite time ruin probability

$$
\psi(x, \alpha)=\mathbb{P}\left[\tau_{x, \alpha}<\infty\right]
$$

where $\tau_{x, \alpha}=\inf \left\{t \geq 0: V_{t}^{x, \alpha}<0\right\}$ is the time of ruin, depending on the initial wealth $x$ and the investment strategy $\alpha$. We are interested in the minimal ruin probability of the insurer

$$
\psi^{*}(x)=\inf _{\alpha \in \mathcal{A}} \psi(x, \alpha)
$$

\subsubsection{Asymptotic ruin probability estimate}

The main result is an asymptotic large deviation estimation for the minimal ruin probability when the initial reserve goes to infinity :

Theorem 3.1 We have

$$
\lim _{x \rightarrow \infty} \frac{1}{x} \ln \psi^{*}(x)=-\theta^{*},
$$

where $\theta^{*}>0$ is he unique positive solution to the equation

$$
\gamma_{Y}(\theta)=p \frac{\theta}{\lambda}+\frac{b^{2}}{2 \sigma^{2} \lambda}
$$

Here $\gamma_{Y}(\theta)=\mathbb{E}\left[e^{\theta Y_{1}}\right]-1$ is the shifted moment generating function of the claim size. Moreover, the constant strategy $\alpha^{*}=\frac{b}{\sigma^{2} \theta^{*}}$ is asymptotically optimal in the sense that

$$
\lim _{x \rightarrow \infty} \frac{1}{x} \ln \psi\left(x, \alpha^{*}\right)=-\theta^{*} .
$$

Finally, if $b \neq 0, \theta^{*}>\theta_{L}$ the Lundberg exponent.

Remark 3.2 The estimate (3.9) is analogue to the classical Lundberg estimate (3.7) without investment. The exponent is larger than the Lundberg one, and thus one gets a sharper bound on the minimal ruin probability. Moreover, the trading strategy yielding the optimal asymptotic exponential decay consists in holding a fixed (explicit) amount in the risky asset. This surprising result, in apparent contradiction with the common believe that 'rich' companies should invest more than 'poor' ones, is explained by the fact that minimization of ruin probability is an extremely conservative criterion. 
We follow the martingale approach of Gerber for stating this theorem. We emphasize the main steps of the proof. Let us introduce, for fixed $x, \theta \in \mathbb{R}_{+}$, and $\alpha \in \mathcal{A}$, the process

$$
M_{t}(x, \theta, \alpha)=\exp \left(-\theta V_{t}^{x, \alpha}\right), \quad t \geq 0 .
$$

Then, a straightforward calculation shows that for any constant process $\alpha=a$, and $t \geq 0$,

$$
\begin{aligned}
\mathbb{E}\left[M_{t}(0, \theta, a)\right] & =\mathbb{E}\left[e^{-\theta\left(p t-\sum_{i=1}^{N_{t}} Y_{i}+a b t+a \sigma W_{t}\right)}\right] \\
& =e^{-\theta(p+a b) t} \mathbb{E}\left[e^{\theta \sum_{i=1}^{N_{t}} Y_{i}}\right] \mathbb{E}\left[e^{-\theta a \sigma W_{t}}\right] \\
& =e^{-\theta(p+a b) t} e^{\gamma_{Y}(\theta) \lambda t} e^{\frac{\theta^{2} a^{2} \sigma^{2}}{2} t} \\
& =e^{f(\theta, a) t},
\end{aligned}
$$

where

$$
f(\theta, a)=\lambda \gamma_{Y}(\theta)-p \theta-a b \theta+\frac{1}{2} a^{2} \theta^{2} \sigma^{2}
$$

We recall that under the assumption of light-tailed distribution on the claim size $Y_{i}$, the shifted moment generating function $\gamma_{Y}$ is finite and convex on $(-\infty, \bar{\theta})$ for some $\bar{\theta} \in(0, \infty]$, and $\gamma_{Y} \rightarrow \infty$ as $\theta$ goes to $\bar{\theta}$. Moreover, recalling that $\mathbb{E}\left[Y_{1}\right] \geq 0$, then $\gamma_{Y}$ is increasing on $[0, \bar{\theta})$ since $\gamma_{Y}^{\prime}(\theta)=\mathbb{E}\left[Y_{1} e^{\theta Y_{1}}\right]>\mathbb{E}\left[Y_{1}\right]$ for $0<\theta<\bar{\theta}$. Now, we see that for all $\theta>0$,

$$
\bar{f}(\theta):=\inf _{a \in \mathbb{R}} f(\theta, a)=\lambda \gamma_{Y}(\theta)-p \theta-\frac{b^{2}}{2 \sigma^{2}},
$$

with an infimum attained for $\hat{a}(\theta)=b /\left(\theta \sigma^{2}\right)$. From the properties of $\gamma_{Y}$, we clearly have the existence and uniqueness of $\theta^{*}$ solution to $\bar{f}\left(\theta^{*}\right)=0$, i.e. (3.10). Since the r.h.s. of (3.10) is just the r.h.s. of (3.4), but shifted by the positive constant $b^{2} / 2 \sigma^{2} \lambda$ (if $b \neq 0$ ), it is also obvious that $\theta^{*}>\theta_{L}$. By choosing $\alpha^{*}=\hat{a}\left(\theta^{*}\right)=b^{2} /\left(\theta^{*} \sigma^{2}\right)$, we then have

$$
\bar{f}\left(\theta^{*}\right)=f\left(\theta^{*}, \alpha^{*}\right)=0 .
$$

A straightforward calculation also shows that for all $a \in \mathbb{R}$,

$$
f\left(\theta^{*}, a\right)=\frac{1}{2}\left(\theta^{*}\right)^{2} \sigma^{2}\left(a-\alpha^{*}\right)^{2} \geq 0 .
$$

Hence, since $V_{t}^{x, \alpha^{*}}$ has independent stationary increments, we obtain similarly as in (3.8), for all $0 \leq t \leq T$,

$$
\begin{aligned}
\mathbb{E}\left[M_{T}\left(x, \theta^{*}, \alpha^{*}\right) \mid \mathcal{F}_{t}\right] & =M_{t}\left(x, \theta^{*}, \alpha^{*}\right) \mathbb{E}\left[M_{T-t}\left(0, \theta^{*}, \alpha^{*}\right)\right] \\
& =M_{t}(x, \theta, a),
\end{aligned}
$$

which shows that the process $M\left(x, \theta^{*}, \alpha^{*}\right)$ is a $(\mathbb{P}, \mathbb{F})$-martingale. Therefore, from the optional sampling theorem at the (bounded) stopping time $\tau_{x, \alpha^{*}} \wedge T$, we have

$$
\begin{aligned}
& e^{-\theta^{*} x}=M_{0}\left(x, \theta^{*}, \alpha^{*}\right)=\mathbb{E}\left[M_{\tau_{x, \alpha^{*} \wedge T}}\left(x, \theta^{*}, \alpha^{*}\right)\right] \\
& =\mathbb{E}\left[M_{\tau_{x, \alpha^{*}}}\left(x, \theta^{*}, \alpha^{*}\right) 1_{\tau_{x, \alpha^{*}} \leq T}\right]+\mathbb{E}\left[M_{T}\left(x, \theta^{*}, \alpha^{*}\right) 1_{\tau_{x, \alpha^{*}>T}}\right] \\
& \geq \mathbb{E}\left[M_{\tau_{x, \alpha^{*}}}\left(x, \theta^{*}, \alpha^{*}\right) 1_{\tau_{x, \alpha^{*} \leq T}}\right] \text {, }
\end{aligned}
$$


since the process $M$ is nonnegative. By the monotone convergence theorem, we then get by sending $T$ to infinity into the previous inequality

$$
e^{-\theta^{*} x} \geq \mathbb{E}\left[M_{\tau_{x, \alpha^{*}}}\left(x, \theta^{*}, \alpha^{*}\right) 1_{\tau_{x, \alpha^{*}}<\infty}\right]=\mathbb{E}\left[M_{\tau_{x, \alpha^{*}}}\left(x, \theta^{*}, \alpha^{*}\right) \mid \tau_{x, \alpha^{*}}<\infty\right] \mathbb{P}\left[\tau_{x, \alpha^{*}}<\infty\right],
$$

from Bayes formula. Thus, we get

$$
\psi\left(x, \alpha^{*}\right)=\mathbb{P}\left[\tau_{x, \alpha^{*}}<\infty\right] \leq \frac{e^{-\theta^{*} x}}{\mathbb{E}\left[M_{\tau_{x, \alpha^{*}}}\left(x, \theta^{*}, \alpha^{*}\right) \mid \tau_{x, \alpha^{*}}<\infty\right]}
$$

Now, by definition of the time of ruin, $V_{\tau_{x, \alpha^{*}}}^{x, \alpha^{*}}$ is nonpositive and so $M_{\tau_{x, \alpha^{*}}}\left(x, \theta^{*}, \alpha^{*}\right) \geq 1$ a.s. on $\left\{\tau_{x, \alpha^{*}}<\infty\right\}$. We deduce that

$$
\psi^{*}(x) \leq \psi\left(x, \alpha^{*}\right) \leq e^{-\theta^{*} x}
$$

In order to state a lower bound on the minimal ruin probability, we proceed as follows. We apply Itô's formula to the process $M\left(x, \theta^{*}, \alpha\right)$ for arbitrary $\alpha \in \mathcal{A}$ :

$$
\frac{d M_{t}\left(x, \theta^{*}, \alpha\right)}{M_{t^{-}}\left(x, \theta^{*}, \alpha\right)}=\left(-\theta^{*}\left(p+b \alpha_{t}\right)+\frac{1}{2}\left(\theta^{*}\right)^{2} \sigma^{2} \alpha_{t}^{2}\right) d t-\theta^{*} \sigma d W_{t}+\left(e^{\theta^{*} Y_{N_{t}}}-1\right) d N_{t} .
$$

Observing that $\gamma_{Y}(\theta)=\mathbb{E}\left[e^{\theta Y_{N_{t}}}-1\right]$, we rewrite as

$$
\begin{aligned}
& \frac{d M_{t}\left(x, \theta^{*}, \alpha\right)}{M_{t^{-}}\left(x, \theta^{*}, \alpha\right)}=\left(-\theta^{*}\left(p+b \alpha_{t}\right)+\frac{1}{2}\left(\theta^{*}\right)^{2} \sigma^{2} \alpha_{t}^{2}+\lambda \gamma_{Y}\left(\theta^{*}\right)\right) d t-\theta^{*} \sigma d W_{t} \\
& +\left(e^{\theta^{*} Y_{N_{t}}}-1\right) d N_{t}-\lambda \mathbb{E}\left[e^{\theta^{*} Y_{N_{t}}}-1\right] d t \\
& =f\left(\theta^{*}, \alpha_{t}\right) d t-\theta^{*} \sigma d W_{t}+d \tilde{N}_{t},
\end{aligned}
$$

where $\tilde{N}_{t}=\int_{0}^{t}\left(e^{\theta^{*} Y_{N_{u}}}-1\right) d N_{u}-\int_{0}^{t} \lambda \mathbb{E}\left[e^{\theta^{*} Y_{N_{u}}}-1\right] d u$. By using the martingale property of $N_{t}-\lambda t$, we can check that $\tilde{N}$ is a martingale. Since $f\left(\theta^{*}, \alpha_{t}\right) \geq 0$ a.s. for all $t$ (see (3.11)), we deduce that $M\left(x, \theta^{*}, \alpha\right)$ is a (local) submartingale. To go to a true submartingale, we need some additional assumption on the distribution of the claim size. Actually, we can prove that under the following uniform exponential tail distribution

$$
\sup _{z \geq 0} \mathbb{E}\left[e^{-\theta^{*}\left(z-Y_{1}\right)} \mid Y_{1}>z\right]=\sup _{z \geq 0} \frac{\int_{z}^{\infty} e^{-\theta^{*}(z-y)} d F_{Y}(y)}{\int_{z}^{\infty} d F_{Y}}<\infty,
$$

the process $M\left(x, \theta^{*}, \alpha\right)$ is an uniformly integrable submartingale. Therefore, from the optional sampling theorem at the (bounded) stopping time $\tau_{x, \alpha} \wedge T$, we have

$$
\begin{aligned}
e^{-\theta^{*} x}=M_{0}\left(x, \theta^{*}, \alpha\right) & \leq \mathbb{E}\left[M_{\tau_{x, \alpha} \wedge T}\left(x, \theta^{*}, \alpha\right)\right] \\
& =\mathbb{E}\left[M_{\tau_{x, \alpha}}\left(x, \theta^{*}, \alpha\right) 1_{\tau_{x, \alpha} \leq T}\right]+\mathbb{E}\left[M_{T}\left(x, \theta^{*}, \alpha\right) 1_{\tau_{x, \alpha}>T}\right]
\end{aligned}
$$

We now claim that $M_{T}\left(x, \theta^{*}, \alpha\right)$ converges a.s. on $\left\{\tau_{x, \alpha}=\infty\right\}$ to zero as $T$ goes to infinity. First, we know from Doob's submartingale convergence theorem that $\lim _{T \rightarrow \infty} M_{T}\left(x, \theta^{*}, \alpha\right)$ exists a.s., hence also $\lim _{T \rightarrow \infty} V_{T}(x, \alpha)$. Since the expectation of a jump size $E\left[Y_{1}\right]$ is positive, there exists $y>0$ s.t. $\mathbb{P}\left[Y_{1}>y\right]>0$. By independence of the jump sizes in the compound Poisson process of the risk reserve, it is then an easy exercice to see that with 
probability 1 , a jump of size greater than $y$ occurs infinitely often on $[0, \infty)$. On the other hand, the stochastic integral due to the invesment strategy $\alpha$ in the stock price, is a.s. continuous, and so cannot compensate the jumps of the compound Poisson process, greater than $y$, which will occur infinitely often a.s. It follows that on $\left\{\tau_{x, \alpha}=\infty\right\}$ (where ruin does not occur), $V_{T}^{x, \alpha}$ cannot converge to a finite value with positive probability. Therefore on $\left\{\tau_{x, \alpha}=\infty\right\}$, we have $\lim _{T \rightarrow \infty} V_{T}(x, \alpha)=\infty$ and thus, since $\theta^{*}>0, \lim _{T \rightarrow \infty} M_{T}\left(x, \theta^{*}, \alpha\right)=$ 0 a.s. As $T \rightarrow \infty$, we have then from the dominated convergence theorem $\left(M_{T}\left(x, \theta^{*}, \alpha\right) \leq\right.$ 1 on $\left\{\tau_{x, \alpha}>T\right\}$ ) for the second term in (3.14), and by the monotone convergence theorem for the first term,

$$
e^{-\theta^{*} x} \leq \mathbb{E}\left[M_{\tau_{x, \alpha}}\left(x, \theta^{*}, \alpha\right) 1_{\tau_{x, \alpha}<\infty}\right]=\mathbb{E}\left[M_{\tau_{x, \alpha}}\left(x, \theta^{*}, \alpha\right) \mid \tau_{x, \alpha}<\infty\right] \mathbb{P}\left[\tau_{x, \alpha}<\infty\right],
$$

and so

$$
\psi(x, \alpha)=\mathbb{P}\left[\tau_{x, \alpha}<\infty\right] \geq \frac{e^{-\theta^{*} x}}{\mathbb{E}\left[M_{\tau_{x, \alpha}}\left(x, \theta^{*}, \alpha\right) \mid \tau_{x, \alpha}<\infty\right]}
$$

We finally prove that $\mathbb{E}\left[M_{\tau_{x, \alpha}}\left(x, \theta^{*}, \alpha\right) \mid \tau_{x, \alpha}<\infty\right]$ is bounded by a constant independent of $\alpha \in \mathcal{A}$. Fix some arbitrary $\alpha \in \mathcal{A}$ and set for shorthand notation $\tau=\tau_{x, \alpha}$ the time of ruin of the wealth process $V^{x, \alpha}$. First observe that ruin $\{\tau<\infty\}$ occurs either through the brownian motion, i.e. on $\left\{\tau<\infty, V_{\tau^{-}}^{x, \alpha}=0\right\}$, and in this case $V_{\tau}^{x, \alpha}=0$ and so $M_{\tau}\left(x, \theta^{*}, \alpha\right)$ $=1$, or through a jump, i.e. on $\left\{\tau<\infty, V_{\tau^{-}}^{x, \alpha}>0\right\}$, and in this case $V_{\tau}^{x, \alpha}<0$ and so $M_{\tau}\left(x, \theta^{*}, \alpha\right)>1$. Hence,

$$
\begin{aligned}
\mathbb{E}\left[M_{\tau}\left(x, \theta^{*}, \alpha\right) \mid \tau<\infty\right] & \leq \mathbb{E}\left[M_{\tau}\left(x, \theta^{*}, \alpha\right) \mid \tau<\infty, V_{\tau^{-}}^{x, \alpha}>0\right] \\
& =\mathbb{E}\left[e^{-\theta^{*}\left(V_{\tau^{-}}^{x, \alpha}-Y_{N_{\tau}}\right)} \mid \tau<\infty, V_{\tau^{-}}^{x, \alpha}>0\right]
\end{aligned}
$$

Let $H^{x, \alpha}(d t, d z)$ denote the joint distribution of $\tau$ and $V_{\tau^{-}}^{x, \alpha}$ conditional on the event $\{\tau<$ $\left.\infty, V_{\tau^{-}}^{x, \alpha}>0\right\}$ that ruin occurs through a jump. Given $\tau=t$ and $V_{\tau^{-}}^{x, \alpha}=z>0$, a claim $Y_{N_{\tau}}$ occurs at time $t$ and has distribution $d F_{Y}(y) / \int_{z}^{\infty} d F_{Y}$ for $y>z$. Hence

$$
\begin{aligned}
\mathbb{E}\left[e^{-\theta^{*}\left(V_{\tau^{-}}^{x, \alpha}-Y_{N_{\tau}}\right)} \mid \tau<\infty, V_{\tau^{-}}^{x, \alpha}>0\right] & =\int_{0}^{\infty} \int_{0}^{\infty} H^{x, \alpha}(d t, d z) \int_{z}^{\infty} e^{-\theta^{*}(z-y)} \frac{d F_{Y}(y)}{\int_{z}^{\infty} d F_{Y}} \\
& \leq \sup _{z \geq 0} \int_{z}^{\infty} e^{-\theta^{*}(z-y)} \frac{d F_{Y}(y)}{\int_{z}^{\infty} d F_{Y}}<\infty
\end{aligned}
$$

by assumption (3.13). By setting

$$
C=\frac{1}{\sup _{z \geq 0} \int_{z}^{\infty} e^{-\theta^{*}(z-y)} \frac{d F_{Y}(y)}{\int_{z}^{\infty} d F_{Y}}}=\inf _{z \geq 0} \frac{\int_{z}^{\infty} d F_{Y}}{\int_{z}^{\infty} e^{-\theta^{*}(z-y)} d F_{Y}(y)} \in(0,1],
$$

we then have from (3.15)-(3.16)-(3.17), for all $x \geq 0$,

$$
\psi(x, \alpha) \geq C e^{-\theta^{*} x}, \quad \forall \alpha \in \mathcal{A} .
$$

Together with the upper bound (3.12), this completes the proof of Theorem 3.1. 


\section{Large deviations and rare event simulation in option pri- cing}

\subsection{Importance sampling and large deviations approximations}

In this paragraph, we show how to use large deviations approximation via importance sampling for Monte-carlo computation of expectations arising in option pricing. In the context of continuous-time models, we are interested in the computation of

$$
I_{g}=\mathbb{E}\left[g\left(S_{t}, 0 \leq t \leq T\right)\right]
$$

where $S$ is the underlying asset price, and $g$ is the payoff of the option, eventually pathdependent, i.e. depending on the path process $S_{t}, 0 \leq t \leq T$. The Monte-Carlo approximation technique consists in simulating $N$ independent sample paths $\left(S_{t}^{i}\right)_{0 \leq t \leq T}, i=1, \ldots, N$, in the distribution of $\left(S_{t}\right)_{0 \leq t \leq T}$, and approximating the required expectation by the sample mean estimator :

$$
I_{g}^{N}=\frac{1}{N} \sum_{i=1}^{N} g\left(S^{i}\right)
$$

The consistency of this estimator is ensured by the law of large numbers, while the error approximation is given by the variance of this estimator from the central limit theorem : the lower is the variance of $g(S)$, the better is the approximation for a given number $N$ of simulations. As already mentioned in the introduction, the basic principle of importance sampling is to reduce variance by changing probability measure from which paths are generated. Here, the idea is to change the distribution of the price process to be simulated in order to take into account the specificities of the payoff function $g$. We focus in this section in the importance sampling technique within the context of diffusion models, and then show how to obtain an optimal change of measure by a large deviation approximation of the required expectation.

\subsubsection{Importance sampling for diffusions via Girsanov's theorem}

We briefly describe the importance sampling variance reduction technique for diffusions. Let $X$ be a $d$-dimensional diffusion process governed by

$$
d X_{s}=b\left(X_{s}\right) d s+\Sigma\left(X_{s}\right) d W_{s},
$$

where $\left(W_{t}\right)_{t \geq 0}$ is a $n$-dimensional brownian motion on a filtered probability space $(\Omega, \mathcal{F}, \mathbb{F}=$ $\left.\left(\mathcal{F}_{t}\right)_{t \geq 0}, \mathbb{P}\right)$, and the Borel functions $b, \Sigma$ satisfy the usual Lipschitz condition ensuring the existence of a strong solution to the s.d.e. (4.1). We denote by $X_{s}^{t, x}$ the solution to (4.1) starting fom $x$ at time $t$, and we define the function :

$$
v(t, x)=\mathbb{E}\left[g\left(X_{s}^{t, x}, t \leq s \leq T\right)\right], \quad(t, x) \in[0, T] \times \mathbb{R}^{d} .
$$

Let $\phi=\left(\phi_{t}\right)_{0 \leq t \leq T}$ be an $\mathbb{R}^{d}$-valued adapted process such that the process

$$
M_{t}=\exp \left(-\int_{0}^{t} \phi_{u}^{\prime} d W_{u}-\frac{1}{2} \int_{0}^{t}\left|\phi_{u}\right|^{2} d u\right), \quad 0 \leq t \leq T,
$$


is a martingale, i.e. $\mathbb{E}\left[M_{T}\right]=1$. This is ensured for instance under the Novikov criterion : $\mathbb{E}\left[\exp \left(\frac{1}{2} \int_{0}^{T}\left|\phi_{u}\right|^{2} d u\right)\right]<\infty$. In this case, one can define a probability measure $\mathbb{Q}$ equivalent to $\mathbb{P}$ on $\left(\Omega, \mathcal{F}_{T}\right)$ by :

$$
\frac{d \mathbb{Q}}{d \mathbb{P}}=M_{T}
$$

Moreover, by Girsanov's theorem, the process $\hat{W}_{t}=W_{t}+\int_{0}^{t} \phi_{u} d u, 0 \leq t \leq T$, is a brownian motion under $\mathbb{Q}$, and the dynamics of $X$ under $\mathbb{Q}$ is given by

$$
d X_{s}=\left(b\left(X_{s}\right)-\Sigma\left(X_{s}\right) \phi_{s}\right) d s+\Sigma\left(X_{s}\right) d \hat{W}_{s} .
$$

From Bayes formula, the expectation of interest can be written as

$$
v(t, x)=\mathbb{E}^{\mathbb{Q}}\left[g\left(X_{s}^{t, x}, t \leq s \leq T\right) L_{T}\right],
$$

where $L$ is the $\mathbb{Q}$-martingale

$$
L_{t}=\frac{1}{M_{t}}=\exp \left(\int_{0}^{t} \phi_{u}^{\prime} d \hat{W}_{u}-\frac{1}{2} \int_{0}^{t}\left|\phi_{u}\right|^{2} d u\right), \quad 0 \leq t \leq T .
$$

The expression (4.3) suggests, for any choice of $\phi$, an alternative Monte-Carlo estimator for $v(t, x)$ with

$$
I_{g, \phi}^{N}(t, x)=\frac{1}{N} \sum_{i=1}^{N} g\left(X^{i, t, x}\right) L_{T}^{i},
$$

by simulating $N$ independent sample paths $\left(X^{i, t, x}\right)$ and $L_{T}^{i}$ of $\left(X^{t, x}\right)$ and $L_{T}$ under $\mathbb{Q}$ given by (4.2)-(4.4). Hence, the change of probability measure through the choice of $\phi$ leads to a modification of the drift process in the simulation of $X$. The variance reduction technique consists in determining a process $\phi$, which induces a smaller variance for the corresponding estimator $I_{g, \phi}$ than the initial one $I_{g, 0}$. The two next paragraphs present two approaches leading to the construction of such processes $\phi$. In the first approach developed in [22], the process $\phi$ is stochastic, and requires an approximation of the expectation of interest. In the second approach due to [25], the process $\phi$ is deterministic and derived through a simple optimization problem. Both approaches rely on asymptotic results from the theory of large deviations.

\subsubsection{Option pricing approximation with a Freidlin-Wentzell large deviation principle}

We are looking for a stochastic process $\phi$, which allows to reduce (possibly to zero!) the variance of the corresponding estimator. The heuristics for achieving this goal is based on the following argument. Suppose for the moment that the payoff $g$ depends only on the terminal value $X_{T}$. Then, by applying Itô's formula to the $\mathbb{Q}$-martingale $v\left(s, X_{s}^{t, x}\right) L_{s}$ between $s=t$ and $s=T$, we obtain :

$$
g\left(X_{T}^{t, x}\right) L_{T}=v(t, x) L_{t}+\int_{t}^{T} L_{s}\left(D_{x} v\left(s, X_{s}^{t, x}\right)^{\prime} \Sigma\left(X_{s}^{t, x}\right)+v\left(x, X_{s}^{t, x}\right) \phi_{s}^{\prime}\right) d \hat{W}_{s} .
$$


Hence, the variance of $I_{g, \phi}^{N}(t, x)$ is given by

$$
\operatorname{Var}_{\mathbb{Q}}\left(I_{g, \phi}^{N}(t, x)\right)=\frac{1}{N} \mathbb{E}^{\mathbb{Q}}\left[\int_{t}^{T} L_{s}^{2}\left|D_{x} v\left(s, X_{s}^{t, x}\right)^{\prime} \Sigma\left(X_{s}^{t, x}\right)+v\left(x, X_{s}^{t, x}\right) \phi_{s}^{\prime}\right|^{2} d s\right] .
$$

The choice of the process $\phi$ is motivated by the following remark. If the function $v$ were known, then one could vanish the variance by choosing

$$
\phi_{s}=\phi_{s}^{*}=-\frac{1}{v\left(s, X_{s}^{t, x}\right)} \Sigma^{\prime}\left(X_{s}^{t, x}\right) D_{x} v\left(s, X_{s}^{t, x}\right), \quad t \leq s \leq T .
$$

Of course, the function $v$ is unknown (this is precisely what we want to compute), but this suggests to use a process $\phi$ from the above formula with an approximation of the function $v$. We may then reasonably hope to reduce the variance, and also to use such a method for more general payoff functions, possibly path-dependent. We shall use a large deviations approximation for the function $v$.

The basic idea for the use of large deviations approximation to the expectation function $v$ is the following. Suppose the option of interest, characterized by its payoff function $g$, has a low probability of exercice, e.g. it is deeply out the money. Then, a large proportion of simulated paths end up out of the exercice domain, giving no contribution to the Montecarlo estimator but increasing the variance. In order to reduce the variance, it is interesting to change of drift in the simulation of price process to make the domain exercice more likely. This is achieved with a large deviations approximation of the process of interest in the asymptotics of small diffusion term : such a result is known in the literature as Freidlin-Wentzell sample path large deviations principle. Equivalently, by time-scaling, this amounts to large deviation approximation of the process in small time, studied by Varadhan.

To illustrate our purpose, let us consider the case of an up-in bond, i.e. an option that pays one unit of numéraire iff the underlying asset reached a given up-barrier $K$. Within a stochastic volatility model $X=(S, Y)$ as in (4.1) and given by :

$$
\begin{aligned}
& d S_{t}=\sigma\left(Y_{t}\right) S_{t} d W_{t}^{1} \\
& d Y_{t}=\eta\left(Y_{t}\right) d t+\gamma\left(Y_{t}\right) d W_{t}^{2}, \quad d<W_{1}, W_{2}>_{t}=\rho d t
\end{aligned}
$$

its price is then given by

$$
v(t, x)=\mathbb{E}\left[1_{\max _{t \leq u \leq T} S_{u}^{t, x} \geq K}\right]=\mathbb{P}\left[\tau_{t, x} \leq T\right], \quad t \in[0, T], x=(s, y) \in(0, \infty) \times \mathbb{R},
$$

where

$$
\tau_{t, x}=\inf \left\{u \geq t: X_{u}^{t, x} \notin \Gamma\right\}, \quad \Gamma=(0, K) \times \mathbb{R} .
$$

The event $\left\{\max _{t \leq u \leq T} S_{u}^{t, x} \geq K\right\}=\left\{\tau_{t, x} \leq T\right\}$ is rare when $x=(s, y) \in \Gamma$, i.e. $s<K$ (out the money option) and the time to maturity $T-t$ is small. The large deviations asymptotics for the exit probability $v(t, x)$ in small time to maturity $T-t$ is provided by the Freidlin-Wentzell and Varadhan theories. Indeed, we see from the time-homogeneity of 
the coefficients of the diffusion and by time-scaling that we may write $v(t, x)=w_{T-t}(0, x)$, where for $\varepsilon>0, w_{\varepsilon}$ is the function defined on $[0,1] \times(0, \infty) \times \mathbb{R}$ by

$$
w_{\varepsilon}(t, x)=\mathbb{P}\left[\tau_{t, x}^{\varepsilon} \leq 1\right]
$$

and $X^{\varepsilon, t, x}$ is the solution to

$$
d X_{s}^{\varepsilon}=\varepsilon b\left(X_{s}^{\varepsilon}\right) d s+\sqrt{\varepsilon} \Sigma\left(X_{s}^{\varepsilon}\right) d W_{s}, \quad X_{t}^{\varepsilon}=x .
$$

and $\tau_{t, x}^{\varepsilon}=\inf \left\{s \geq t: X_{s}^{\varepsilon, t, x} \notin \Gamma\right\}$. From the large deviations result (2.19) stated in paragraph 2.3, we have :

$$
\lim _{t \nearrow T}-(T-t) \ln v(t, x)=V_{0}(0, x)
$$

where

$$
\begin{gathered}
V_{0}(t, x)=\inf _{x(.) \in \mathcal{A}(t, x)} \int_{t}^{1} \frac{1}{2} \dot{x}(u)^{\prime} M(x(u)) \dot{x}(u) d u, \quad(t, x) \in[0,1) \times \Gamma, \\
\Sigma(x)=\left(\begin{array}{cc}
\sigma(x) & 0 \\
0 & \gamma(x)
\end{array}\right) \text { is the diffusion matrix of } X=(S, Y), M(x)=\left(\Sigma \Sigma^{\prime}(x)\right)^{-1}, \text { and } \\
\mathcal{A}(t, x)=\left\{x(.) \in H_{l o c}^{1}([0,1],(0, \infty) \times \mathbb{R}): x(t)=x \text { and } \tau(x) \leq 1\right\} .
\end{gathered}
$$

Here, for an absolutely continuous function $x($.$) on [0,1)$ and valued in $(0, \infty) \times \mathbb{R}$, we denote $\dot{x}(u)$ its time derivative, and $\tau(x)$ the exit time of $x($.$) from \Gamma$.

We also have another interpretation of the positive function $V_{0}$ in terms of Riemanian distance on $\mathbb{R}^{d}$ associated to the metric $M(x)=\left(\Sigma \Sigma^{\prime}(x)\right)^{-1}$. By denoting $L_{0}(x)=$ $\sqrt{2 V_{0}(0, x)}$, one can prove (see [34]) that $L_{0}$ is the unique viscosity solution to the eikonal equation

$$
\begin{aligned}
\left(D_{x} L_{0}\right)^{\prime} \Sigma \Sigma^{\prime}(x) D_{x} L_{0} & =1, \quad x \in \Gamma \\
L_{0}(x) & =0, \quad x \in \partial \Gamma
\end{aligned}
$$

and that it may be represented as

$$
L_{0}(x)=\inf _{z \in \partial \Gamma} L_{0}(x, z), \quad x \in \Gamma
$$

where

$$
L_{0}(x, z)=\inf _{x(.) \in A(x, z)} \int_{0}^{1} \sqrt{\dot{x}(u)^{\prime} M(x(u)) \dot{x}(u)} d u
$$

and $A(x, z)=\left\{x(.) \in H_{l o c}^{1}([0,1],(0, \infty) \times \mathbb{R}): x(0)=x\right.$ and $\left.x(1)=z\right\}$. Hence, the function $L_{0}$ can be computed either by the numerical resolution of the eikonal equation or by using the representation (4.8). $L_{0}(x)$ is interpreted as the minimal length (according to the metric $M$ ) of the path $x($.$) allowing to reach the boundary \partial \Gamma$ from $x$. From the above large deviations result, which is written as

$$
\ln v(t, x) \simeq-\frac{L_{0}^{2}(x)}{2(T-t)}, \quad \text { as } \quad T-t \rightarrow 0,
$$


and the expression (4.5) for the optimal theoretical $\phi^{*}$, we use a change of probability measure with

$$
\phi(t, x)=\frac{L_{0}(x)}{T-t} \Sigma^{\prime}(x) D_{x} L_{0}(x) .
$$

Such a process $\phi$ may also appear interesting to use in a more general framework than up-in bond : one can use it for computing any option whose exercice domain looks similar to the up and in bond. We also expect that the variance reduction is more significant as the exercice probability is low, i.e. for deep out the money options. In the particular case of the Black-Scholes model, i.e. $\sigma(x) \sigma s$, we have

$$
L_{0}(x)=\frac{1}{\sigma}\left|\ln \left(\frac{s}{K}\right)\right|,
$$

and so

$$
\phi(t, x)=\frac{1}{\sigma(T-t)} \ln \left(\frac{s}{K}\right), \quad s<K .
$$

\subsubsection{Change of drift via Varadhan-Laplace principle}

We describe here a method due to [25], which, in contrast with the above approach, does not require the knowledge of the option price. This method restricts to deterministic changes of drift over discrete time steps. Hence, the diffusion model $X$ for state variables (stock price, volatility) is simulated (eventually using an Euler scheme if needed) on a discrete time grid $0=t_{0}<t_{1}<\ldots<t_{m}=T$ : the increment of the brownian motion from $t_{i-1}$ to $t_{i}$ is simulated as $\sqrt{t_{i}-t_{i-1}} Z_{i}$, where $Z_{1}, \ldots, Z_{m}$ are i.i.d. $n$-dimensional standard normal random vectors. We denote by $Z$ the concatenation of the $Z_{i}$ into a single vector of lengh $l$ $=m n$. Each outcome of $Z$ determines a path of state variables. Let $G(Z)$ denote the payoff derived from $Z$, and our aim is to compute the (path-dependent) option price $\mathbb{E}[G(Z)]$. For example, in the case of the Black-Scholes model for the stock price $S$, we have

$$
S_{t_{i}}=S_{t_{i-1}} \exp \left(-\frac{\sigma^{2}}{2}\left(t_{i}-t_{i-1}\right)+\sigma \sqrt{t_{i}-t_{i-1}} Z_{i}\right)
$$

and the payoff of the Asian option is

$$
G(Z)=G\left(Z_{1}, \ldots, Z_{m}\right)=\left(\frac{1}{m} \sum_{i=1}^{m} S_{t_{i}}-K\right)_{+}
$$

We apply importance sampling by changing the mean of $Z$ from 0 to some vector $\mu=$ $\left(\mu_{1}, \ldots, \mu_{m}\right)$. We denote $\mathbb{P}_{\mu}$ and $\mathbb{E}_{\mu}$ the probability and expectation when $Z \sim \mathcal{N}\left(\mu, I_{m}\right)$. Notice that with the notations of paragraph 4.1.1, this corresponds to a piecewise constant process $\phi$ s.t. $\phi_{t}=-\mu_{i} / \sqrt{t_{i}-t_{i-1}}$ on $\left[t_{i-1}, t_{i}\right)$. By Girsanov's theorem or here more simply from the likelihood ratio for normal random vectors, the corresponding unbiased estimator is then obtained by taking the average of independent replications of

$$
\vartheta_{\mu}=G(Z) e^{-\mu^{\prime} Z+\frac{1}{2} \mu^{\prime} \mu}
$$


where $Z$ is sampled from $\mathcal{N}\left(\mu, I_{m}\right)$. We call $\vartheta_{\mu}$ a $\mu$-IS estimator. In order to minimize over $\mu$ the variance of this estimator, it suffices to minimize its second moment, which is given by :

$$
M^{2}(\mu)=\mathbb{E}_{\mu}\left[G(Z)^{2} e^{-2 \mu^{\prime} Z+\mu^{\prime} \mu}\right]=\mathbb{E}\left[G(Z)^{2} e^{-\mu^{\prime} Z+\frac{1}{2} \mu^{\prime} \mu}\right]
$$

We are then looking for an optimal $\mu$ solution to

$$
\inf _{\mu} M^{2}(\mu):=\inf _{\mu} \mathbb{E}\left[G(Z)^{2} e^{-\mu^{\prime} Z+\frac{1}{2} \mu^{\prime} \mu}\right] .
$$

This minimization problem is, in general, a well-posed problem. Indeed, it is shown in [2] that if $\mathbb{P}[G(Z)>0]>0$, and $\mathbb{E}\left[G(Z)^{2+\delta}\right]<\infty$ for some $\delta>0$, then $M^{2}($.$) is a strictly convex$ function, and thus $\mu^{*}$ solution to (4.9) exists and is unique. This $\mu^{*}$ can be computed by solving (numerically) $\nabla M^{2}(\mu)=0$. This is the method adopted in [2] with a RobbinsMonro stochastic algorithm. We present here an approximate resolution of (4.9) by means of large deviations approximation. For this, assume that $G$ takes only nonnegative values, so that it is written as $G(z)=\exp (F(z))$, with the convention that $F(z)=-\infty$ if $G(z)=$ 0 , and let us consider the more general estimation problem where $Z$ is replaced by $Z^{\varepsilon}=$ $\sqrt{\varepsilon} Z$ and we simultaneously scale the payoff by raising it to the power of $1 / \varepsilon$ :

$$
v_{\varepsilon}=\mathbb{E}\left[e^{\frac{1}{\varepsilon} F\left(Z^{\varepsilon}\right)}\right]
$$

The quantity of interest $\mathbb{E}[G(Z)]=\mathbb{E}\left[e^{F(Z)}\right]$ is $v_{\varepsilon}$ for $\varepsilon=1$. We embed the problem of estimating $v_{1}$ in the more general problem of estimating $v_{\varepsilon}$ and analyze the second moment of corresponding IS estimators as $\varepsilon$ is small, by means of Varadhan-Laplace principle. For any $\mu$, we consider $\mu_{\varepsilon}$-IS estimator of $v_{\varepsilon}$ with $\mu^{\varepsilon}=\mu / \sqrt{\varepsilon}$ :

$$
\vartheta_{\mu}^{\varepsilon}=e^{\frac{1}{\varepsilon} F(\sqrt{\varepsilon} Z)} e^{-\mu_{\varepsilon}^{\prime} Z+\frac{1}{2} \mu_{\varepsilon}^{\prime} \mu_{\varepsilon}}=e^{\frac{1}{\varepsilon}\left(F\left(Z^{\varepsilon}\right)-\mu^{\prime} Z^{\varepsilon}+\frac{1}{2} \mu^{\prime} \mu\right)}
$$

where $Z$ is sampled from $\mathcal{N}\left(\mu_{\varepsilon}, I_{m}\right)=\mathcal{N}\left(\mu / \sqrt{\varepsilon}, I_{m}\right)$. Its second moment is

$$
\left.M_{\varepsilon}^{2}(\mu)=\mathbb{E}_{\mu_{\varepsilon}}\left[e^{\frac{1}{\varepsilon}\left(2 F\left(Z^{\varepsilon}\right)-2 \mu^{\prime} Z^{\varepsilon}+\mu^{\prime} \mu\right)}\right]=\mathbb{E}\left[e^{\frac{1}{\varepsilon}\left(2 F\left(Z^{\varepsilon}\right)-\mu^{\prime} Z^{\varepsilon}+\frac{1}{2} \mu^{\prime} \mu\right.}\right)\right]
$$

Now, from Cramer's theorem, $\left(Z^{\varepsilon}\right)_{\varepsilon}$ satisfies a LDP with rate function $I(z)=\frac{1}{2} z^{\prime} z$. Hence, under the condition that $F(z) \leq c_{1}+c_{2} z^{\prime} z$ for some $c_{2}<1 / 4$, one can apply Varadhan's integral principle (see Theorem 2.2) to the function $z \rightarrow 2 F(z)-\mu^{\prime} z+\frac{1}{2} \mu^{\prime} \mu$, and get

$$
\lim _{\varepsilon \rightarrow 0} \varepsilon \ln M_{\varepsilon}^{2}(\mu)=\sup _{z}\left[2 F(z)-\mu^{\prime} z+\frac{1}{2} \mu^{\prime} \mu-\frac{1}{2} z^{\prime} z\right] .
$$

This suggests to search for a $\mu$ solution to the problem :

$$
\inf _{\mu} \sup _{z}\left[2 F(z)-\mu^{\prime} z+\frac{1}{2} \mu^{\prime} \mu-\frac{1}{2} z^{\prime} z\right] .
$$

This min-max problem may be reduced to a simpler one. Indeed, assuming that the conditions of the min/max theorem hold, then the inf and sup can be permuted, and we find

$$
\begin{aligned}
\inf _{\mu} \sup _{z}\left[2 F(z)-\mu^{\prime} z+\frac{1}{2} \mu^{\prime} \mu-\frac{1}{2} z^{\prime} z\right] & =\sup _{z}\left[\inf _{\mu}\left(-\mu^{\prime} z+\frac{1}{2} \mu^{\prime} \mu\right)+2 F(z)-\frac{1}{2} z^{\prime} z\right] \\
& =2 \sup _{z}\left[F(z)-\frac{1}{2} z^{\prime} z\right] .
\end{aligned}
$$


Actually, under suitable convexity conditions on $F$ and its domain, one can show (see [25]) that (4.12) holds. Furthermore, if $\hat{z}$ is solution to

$$
\sup _{z}\left(F(z)-\frac{1}{2} z^{\prime} z\right)
$$

then a solution $\hat{\mu}$ solution to (4.11) should be identified with the conjugate of $\hat{z}$, via $\inf _{\mu}\left(-\mu^{\prime} \hat{z}+\frac{1}{2} \mu^{\prime} \mu\right)$ that is $\hat{\mu}=\hat{z}$. The solution to problem (4.13) has also the following interpretation. From heuristic arguments of importance sampling (see the introduction), an optimal effective importance sampling density should assign high probability to regions on which the product of the integrand payoff and the original density is large. For our problem, this product is proportional to

$$
e^{F(z)-\frac{1}{2} z^{\prime} z}
$$

since $\exp \left(-z^{\prime} z / 2\right)$ is proportional to the standard normal density. This suggests to choose $\mu=\hat{\mu}$ solution to (4.13). Another heuristics indication for the choice of (4.13) is based on the following argument. Assume that $F$ is $C^{1}$ on its domain, and the maximum $\hat{\mu}$ in (4.13) is attained in the interior of the domain, so that it solves the fixed point equation :

$$
\nabla F(\hat{\mu})=\hat{\mu} .
$$

By using a first-order Taylor approximation of $F$ around the mean $\hat{\mu}$ of $Z$ under $\mathbb{P}_{\hat{\mu}}$, we may approximate the estimator as

$$
\vartheta_{\hat{\mu}}=e^{F(Z)-\hat{\mu}^{\prime} Z+\frac{1}{2} \hat{\mu}^{\prime} \hat{\mu}} \simeq e^{F(\hat{\mu})+\nabla F(\hat{\mu})^{\prime}(Z-\hat{\mu})-\hat{\mu}^{\prime} Z+\frac{1}{2} \hat{\mu}^{\prime} \hat{\mu}}
$$

Hence, for the choice of $\hat{\mu}$ satisfying (4.14), the expression of the r.h.s. of (4.15) collapses to a constant with no dependence on $Z$. Thus, applying importance sampling with such a $\hat{\mu}$ would produce a zero-variance estimator if (4.15) holds exactly, e.g. if $F$ is linear, and it should produce a low-variance estimator if (4.15) holds only approximately.

The choice of $\mu=\hat{\mu}$ solution to (4.13) leads also to an asymptotically optimal ISestimator in the following sense. First, notice that for any $\mu$, we have by Cauchy-Schwarz's inequality : $M_{\varepsilon}^{2}(\mu) \geq\left(v_{\varepsilon}\right)^{2}$, and so

$$
\lim _{\varepsilon \rightarrow 0} \varepsilon \ln M_{\varepsilon}^{2}(\mu) \geq 2 \lim _{\varepsilon \rightarrow 0} \varepsilon \ln v_{\varepsilon}=2 \lim _{\varepsilon \rightarrow 0} \varepsilon \ln \mathbb{E}\left[e^{\frac{1}{\varepsilon} F\left(Z^{\varepsilon}\right)}\right]
$$

From Varadhan's integral principle applied to the function $z \rightarrow F(z)$, we thus deduce for any $\mu$,

$$
\lim _{\varepsilon \rightarrow 0} \varepsilon \ln M_{\varepsilon}^{2}(\mu) \geq 2 \sup _{z}\left[F(z)-\frac{1}{2} z^{\prime} z\right]=2\left[F(\hat{\mu})-\frac{1}{2} \hat{\mu}^{\prime} \hat{\mu}\right] .
$$

Hence, $2\left[F(\hat{\mu})-\frac{1}{2} \hat{\mu}^{\prime} \hat{\mu}\right]$ is the best-possible exponential decay rate for a $\mu_{\varepsilon}$-IS estimator $\vartheta_{\mu}^{\varepsilon}$. Now, by choosing $\mu=\hat{\mu}$, and from (4.10), (4.12), we have

$$
\lim _{\varepsilon \rightarrow 0} \varepsilon \ln M_{\varepsilon}^{2}(\hat{\mu})=2\left[F(\hat{\mu})-\frac{1}{2} \hat{\mu}^{\prime} \hat{\mu}\right]
$$

which shows that the $\hat{\mu}_{\varepsilon}$-IS estimator $\vartheta_{\hat{\mu}}^{\varepsilon}$ is asymptotically optimal. 
Remark 4.1 From the first-order approximation of $F$ in (4.15), we see that in order to obtain further variance reduction, it is natural to address the quadratic component of $F$. This can be achieved by a method of stratified sampling as developed in [25].

Recently, the above approach of [25] was extended in [29] to a continuous-time setting, where the optimal deterministic drift in the Black-Scholes model is characterized as the solution to a classical one-dimensional variational problem.

\subsection{Computation of barrier crossing probabilities and sharp large devia- tions}

In this paragraph, we present a simulation procedure for computing the probability that a diffusion process crosses pre-specified barriers in a given time interval $[0, T]$. Let $\left(X_{t}\right)_{t \in[0, T]}$ be a diffusion process in $\mathbb{R}^{d}$,

$$
d X_{t}=b\left(X_{t}\right) d t+\sigma\left(X_{t}\right) d W_{t}
$$

and $\tau$ is the exit time of $X$ from some domain $\Gamma$ of $\mathbb{R}^{d}$, eventually depending on time :

$$
\tau=\inf \left\{t \in[0, T]: X_{t} \notin \Gamma(t)\right\}
$$

with the usual convention that $\inf \emptyset=\infty$. Such a quantity appears typically in finance in the computation of barrier options, for example with a knock-out option :

$$
C_{0}=\mathbb{E}\left[e^{-r T} g\left(X_{T}\right) 1_{\tau>T}\right],
$$

with $\Gamma(t)=(-\infty, U(t))$ in the case of single barrier options, and $\Gamma(t)=(L(t), U(t))$, for double barrier options. Here, $L, U$ are real functions : $[0, \infty) \rightarrow(0, \infty)$ s.t. $L<U$.

The direct naive approach would consist first of simulating the process $X$ on $[0, T]$ through a discrete Euler scheme of step size $\varepsilon=T / n=t_{i+1}-t_{i}, i=0, \ldots, n$ :

$$
\bar{X}_{t_{i}+1}^{\varepsilon}=\bar{X}_{t_{i}}^{\varepsilon}+b\left(\bar{X}_{t_{i}}^{\varepsilon}\right) \varepsilon+\sigma\left(\bar{X}_{t_{i}}^{\varepsilon}\right)\left(W_{t_{i+1}}-W_{t_{i}}\right),
$$

and the exit time $\tau$ is approximated by the first time the discretized process reaches the barrier :

$$
\bar{\tau}^{\varepsilon}=\inf \left\{t_{i}: \bar{X}_{t_{i}}^{\varepsilon} \notin \Gamma\left(t_{i}\right)\right\} .
$$

Then, the barrier option price $C_{0}$ in (4.16) is approximated by Monte-Carlo simulations of the quantity

$$
\bar{C}_{0}^{\varepsilon}=\mathbb{E}\left[e^{-r T} g\left(\bar{X}_{T}^{\varepsilon}\right) 1_{\bar{\tau}^{\varepsilon}>T}\right] .
$$

In this procedure, one considers that the price diffusion is killed if there exists a value $\bar{X}_{t_{i}}^{\varepsilon}$, which is out of the domain $\Gamma\left(t_{i}\right)$. Hence, such an approach is suboptimal since it does not control the diffusion path between two successive dates $t_{i}$ and $t_{i+1}$ : the diffusion path could have crossed the barriers and come back to the domain without being detected. In this case, one over-estimates the exit time probability of $\{\tau>T\}$. This suboptimality is 
confirmed by the property that the error between $C_{0}$ and $\bar{C}_{0}^{\varepsilon}$ is of order $\sqrt{\varepsilon}$, as shown in [28], instead of the usual order $\varepsilon$ obtained for standard vanilla options.

In order to improve the above procedure, we need to determine the probability that the process $X$ crosses the barrier between discrete simulation times. We then consider the continuous Euler scheme

$$
\bar{X}_{t}^{\varepsilon}=\bar{X}_{t_{i}}^{\varepsilon}+b\left(\bar{X}_{t_{i}}^{\varepsilon}\right)\left(t-t_{i}\right)+\sigma\left(\bar{X}_{t_{i}}^{\varepsilon}\right)\left(W_{t}-W_{t_{i}}\right), \quad t_{i} \leq t \leq t_{i+1},
$$

which evolves as a Brownian with drift between two time discretizations $t_{i}, t_{i+1}=t_{i}+\varepsilon$. Given a simulation path of $\left(\bar{X}_{t_{i}}^{\varepsilon}\right)_{i}$, and values $\bar{X}_{t_{i}}^{\varepsilon}=x_{i}, \bar{X}_{t_{i+1}}^{\varepsilon}=x_{i+1}$, we denote

$$
p_{i}^{\varepsilon}\left(x_{i}, x_{i+1}\right)=\mathbb{P}\left[\exists t \in\left[t_{i}, t_{i+1}\right]: \bar{X}_{t}^{\varepsilon} \notin \Gamma\left(t_{i}\right) \mid\left(\bar{X}_{t_{i}}^{\varepsilon}, \bar{X}_{t_{i+1}}^{\varepsilon}\right)=\left(x_{i}, x_{i+1}\right)\right],
$$

the exit probability of the Euler scheme conditionally on the simulated path values. The correction Monte-Carlo procedure works then as follows : with probability $p_{i}^{\varepsilon}=p_{i}^{\varepsilon}\left(\bar{X}_{t_{i}}^{\varepsilon}, \bar{X}_{t_{i+1}}^{\varepsilon}\right)$, we stop the simulation by considering that the diffusion is killed, and we set $\tau^{\varepsilon}=t_{i}$; with probability $1-p_{i}^{\varepsilon}$, we carry on the simulation. The approximation of (4.16) is thus computed by Monte-Carlo simulations of

$$
C_{0}^{\varepsilon}=\mathbb{E}\left[e^{-r T}\left(\bar{X}_{T}^{\varepsilon}-K\right)_{+} 1_{\tau^{\varepsilon}>T}\right] .
$$

We then recover a rate of convergence of order $\varepsilon$ for $C_{0}^{\varepsilon}-C_{0}$, see [28].

The effective implementation of this corrected procedure requires the calculation of $p_{i}^{\varepsilon}$. Notice that on the interval $\left[t_{i}, t_{i+1}\right]$, the diffusion $\bar{X}^{\varepsilon}$ conditionned to $\bar{X}_{t_{i}}^{\varepsilon}=x_{i}, \bar{X}_{t_{i+1}}^{\varepsilon}=$ $x_{i+1}$, is a brownian bridge : it coincides in distribution with the process

$$
\tilde{B}_{t}^{i, \varepsilon}=x_{i}+\frac{t}{\varepsilon}\left(x_{i+1}-x_{i}\right)+\sigma\left(x_{i}\right)\left(W_{t}-\frac{t}{\varepsilon} W_{\varepsilon}\right), \quad 0 \leq t \leq \varepsilon,
$$

and so by time change $t \rightarrow t / \varepsilon$, with the process

$$
Y_{t}^{i, \varepsilon}:=\tilde{B}_{\varepsilon t}^{i, \varepsilon}=x_{i}+t\left(x_{i+1}-x_{i}\right)+\sqrt{\varepsilon} \sigma\left(x_{i}\right)\left(W_{t}-t W_{1}\right), \quad 0 \leq t \leq 1 .
$$

It is known that the process $Y^{i, \varepsilon}$ is solution to the s.d.e.

$$
\begin{aligned}
d Y_{t}^{i, \varepsilon} & =-\frac{Y_{t}^{i, \varepsilon}-x_{i+1}}{1-t} d t+\sqrt{\varepsilon} \sigma\left(x_{i}\right) d W_{t}, \quad 0 \leq t<1, \\
Y_{0}^{i, \varepsilon} & =x_{i} .
\end{aligned}
$$

The probability $p_{i}^{\varepsilon}$ can then be expressed as

$$
p_{i}^{\varepsilon}\left(x_{i}, x_{i+1}\right)=\mathbb{P}\left[\tau^{i, \varepsilon} \leq 1\right], \quad \text { where } \tau^{i, \varepsilon}=\inf \left\{t \geq 0: Y_{t}^{i, \varepsilon} \notin \Gamma\left(t_{i}+\varepsilon t\right)\right\} .
$$

In the case of a half-space, i.e. single constant barrier, one has an explicit expression for the exit probability of a Brownian bridge. For example, if $\Gamma(t)=(-\infty, U)$, we have

$p_{i}^{\varepsilon}\left(x_{i}, x_{i+1}\right)=\exp \left(-\frac{I_{U}\left(x_{i}, x_{i+1}\right)}{\varepsilon}\right), \quad$ with $I_{U}\left(x_{i}, x_{i+1}\right)=\frac{2}{\sigma^{2}\left(x_{i}\right)}\left(U-x_{i}\right)\left(U-x_{i+1}\right)$. 
In the general case, we do not have analytical expressions for $p_{i}^{\varepsilon}$, and one has to rely on simulation techniques or asymptotic approximations. We shall here consider asymptotic techniques based on large deviations and Freidlin-Wentzell theory. Let us illustrate this point in the case of two time-dependent barriers, i.e. $\Gamma(t)=(L(t), U(t))$ for smooth barriers functions $L<U$. Problem (4.17) does not exactly fit into the Freidlin-Wentzell framework considered in paragraph 2.3, but was adapted for Brownian bridges with time-dependent barriers in [7]. We then have the large deviation estimate for $p_{i}^{\varepsilon}$ :

$$
\lim _{\varepsilon \rightarrow 0} \varepsilon \ln p_{i}^{\varepsilon}\left(x_{i}, x_{i+1}\right)=-I_{L, U}\left(x_{i}, x_{i+1}\right),
$$

where $I_{L, U}\left(x_{i}, x_{i+1}\right)$ is the infimum of the functional

$$
y(.) \longrightarrow \frac{1}{2 \sigma\left(x_{i}\right)^{2}} \int_{0}^{1}\left|\dot{y}(t)+\frac{y(t)-x_{i+1}}{1-t}\right|^{2} d t,
$$

over all absolutely continuous paths $y($.$) on [0,1]$ s.t. $y(0)=x_{i}$, and there exists some $t \in$ $[0,1]$ for which $y(t) \leq L\left(t_{i}\right)$ or $y(t) \geq U\left(t_{i}\right)$. This infimum is a classical problem of calculus of variations, which is explicitly solved and gives for any $x_{i}, x_{i+1} \in\left(L\left(t_{i}\right), U\left(t_{i}\right)\right)$ (otherwise $\left.I_{L, U}\left(x_{i}, x_{i+1}\right)=0\right)$ :

$$
I_{L, U}\left(x_{i}, x_{i+1}\right)= \begin{cases}\frac{2}{\sigma^{2}\left(x_{i}\right)}\left(U\left(t_{i}\right)-x_{i}\right)\left(U\left(t_{i}\right)-x_{i+1}\right) & \text { if } x_{i}+x_{i+1}>L\left(t_{i}\right)+U\left(t_{i}\right) \\ \frac{2}{\sigma^{2}\left(x_{i}\right)}\left(x_{i}-L\left(t_{i}\right)\right)\left(x_{i+1}-L\left(t_{i}\right)\right) & \text { if } x_{i}+x_{i+1}<L\left(t_{i}\right)+U\left(t_{i}\right) .\end{cases}
$$

In order to remove the log estimate on $p_{i}^{\varepsilon}$, we need a sharper large deviation estimate, and this is analyzed by the results of [17] recalled in paragraph 2.3. More precisely, we have

$$
p_{i}^{\varepsilon}\left(x_{i}, x_{i+1}\right)=\exp \left(-\frac{I_{L, U}\left(x_{i}, x_{i+1}\right)}{\varepsilon}-w_{L, U}\left(x_{i}, x_{i+1}\right)\right)(1+O(\varepsilon)),
$$

where $w_{L, U}\left(x_{i}, x_{i+1}\right)$ is explicited in [7] as

$$
w_{L, U}\left(x_{i}, x_{i+1}\right)= \begin{cases}\frac{2}{\sigma^{2}\left(x_{i}\right)}\left(U\left(t_{i}\right)-x_{i}\right) U^{\prime}\left(t_{i}\right) & \text { if } x_{i}+x_{i+1}>L\left(t_{i}\right)+U\left(t_{i}\right) \\ \frac{2}{\sigma^{2}\left(x_{i}\right)}\left(x_{i}-L\left(t_{i}\right)\right) L^{\prime}\left(t_{i}\right) & \text { if } x_{i}+x_{i+1}<L\left(t_{i}\right)+U\left(t_{i}\right) .\end{cases}
$$

Some recent extensions of this large deviations approach to the computation of exit probabilities for multivariate Brownian bridge are studied in [32], which also gives applications for the estimation of default probabilities in credit risk models, and the pricing of credit default swaps.

\section{$5 \quad$ Large deviations in risk management}

\subsection{Large portfolio losses in credit risk}

\subsubsection{Portfolio credit risk in a single factor normal copula model}

A basic problem in measuring portfolio credit risk is determining the distribution of losses from default over a fixed horizon. Credit portfolios are often large, including exposure to thousands of obligors, and the default probabilities of high-quality credits are extremely 
small. These features in credit risk context lead to consider rare but significant large loss events, and emphasis is put on the small probabilities of large losses, as these are relevant for calculation of value at risk and related risk measures.

We use the following notation :

$n=$ number of obligors to which portfolio is exposed,

$Y_{k}=$ default indicator ( $=1$ if default, 0 otherwise) for $k$-th obligor,

$p_{k}=$ marginal probability that $k$-th obligor defaults, i.e. $p_{k}=\mathbb{P}\left[Y_{k}=1\right]$,

$c_{k}=$ loss resulting from default of the $k$-th obligor,

$L_{n}=c_{1} Y_{1}+\ldots+c_{n} Y_{n}=$ total loss from defaults.

We are interested in estimating tail probabilities $\mathbb{P}\left[L_{n}>\ell_{n}\right]$ in the limiting regime at increasingly high loss thresholds $\ell_{n}$, and rarity of large losses resulting from a large number $n$ of obligors and multiple defaults.

For simplicity, we consider a homogeneous portfolio where all $p_{k}$ are equal to $p$, and all $c_{k}$ are equal constant to 1 . An essential feature for credit risk management is the mechanism used to model the dependence across sources of credit risk. The dependence among obligors is modelled by the dependence among the default indicators $Y_{k}$. This dependence is introduced through a normal copula model as follows : each default indicator is represented as

$$
Y_{k}=1_{\left\{X_{k}>x_{k}\right\}}, \quad k=1, \ldots, n,
$$

where $\left(X_{1}, \ldots, X_{n}\right)$ is a multivariate normal vector. Without loss of generality, we take each $X_{k}$ to have a standard normal distribution, and we choose $x_{k}$ to match the marginal default probability $p_{k}$, i.e. $x_{k}=\Phi^{-1}\left(1-p_{k}\right)=-\Phi^{-1}\left(p_{k}\right)$, with $\Phi$ cumulative normal distribution. We also denote $\varphi=\Phi^{\prime}$ the density of the normal distribution. The correlations along the $X_{k}$, which determine the dependence among the $Y_{k}$, are specified through a single factor model of the form :

$$
X_{k}=\rho Z+\sqrt{1-\rho^{2}} \varepsilon_{k}, \quad k=1, \ldots, n .
$$

where $Z$ has the standard normal distribution $\mathcal{N}(0,1), \varepsilon_{k}$ are independent $\mathcal{N}(0,1)$ distribution, and $Z$ is independent of $\varepsilon_{k}, k=1, \ldots, n$. $Z$ is called systematic risk factor (industry, regional risk factors for example $\ldots)$, and $\varepsilon_{k}$ is an idiosyncratic risk associated with the $k$-th obligor. The constant $\rho$ in $[0,1)$ is a factor loading on the single factor $Z$, and assumed here to be identical for all obligors. We shall distinguish the case of independent obligors $(\rho=0)$, and dependent obligors $(\rho>0)$. More general multivariate factor models with inhomogeneous obligors are studied in [26].

\subsubsection{Independent obligors}

In this case, $\rho=0$, the default indicators $Y_{k}$ are i.i.d. with Bernoulli distribution of parameter $p$, and $L_{n}$ is a binomial distribution of parameters $n$ and $p$. By the law of large numbers, $L_{n} / n$ converges to $p$. Hence, in order that the loss event $\left\{L_{n} \geq l_{n}\right\}$ becomes rare (without being trivially impossible), we let $l_{n} / n$ approach $q \in(p, 1)$. It is then appropriate 
to specify $l_{n}=n q$ with $p<q<1$. From Cramer's theorem and the expressions of the c.g.f. of the Bernoulli distribution and its Fenchel-Legendre transform, we obtain the large deviation result for the loss probability :

$$
\lim _{n \rightarrow} \frac{1}{n} \ln \mathbb{P}\left[L_{n} \geq n q\right]=-q \ln \left(\frac{q}{p}\right)-(1-q) \ln \left(\frac{1-q}{1-p}\right)<0 .
$$

Remark 5.1 By denoting $\Gamma(\theta)=\ln \left(1-p+p e^{\theta}\right)$ the c.g.f. of $Y_{k}$, we have an IS (unbiased) estimator of $\mathbb{P}\left[L_{n} \geq n q\right]$ by taking the average of independent replications of

$$
\exp \left(-\theta L_{n}+n \Gamma(\theta)\right) 1_{L_{n} \geq n q}
$$

where $L_{n}$ is sampled with a default probability $p(\theta)=\mathbb{P}_{\theta}\left[Y_{k}=1\right]=p e^{\theta} /\left(1-p+p e^{\theta}\right)$. Moreover, see Remark 2.2, this estimator is asymptotically optimal, as $n$ goes to infinity, for the choice of parameter $\theta_{q} \geq 0$ attaining the argmax in $\theta q-\Gamma(\theta)$.

\subsubsection{Dependent obligors}

We consider the case where $\rho>0$. Then, conditionally on the factor $Z$, the default indicators $Y_{k}$ are i.i.d. with Bernoulli distribution of parameter :

$$
\begin{aligned}
p(Z) & =\mathbb{P}\left[Y_{k}=1 \mid Z\right]=\mathbb{P}\left[\rho Z+\sqrt{1-\rho^{2}} \varepsilon_{k}>-\Phi^{-1}(p) \mid Z\right] \\
& =\Phi\left(\frac{\rho Z+\Phi^{-1}(p)}{\sqrt{1-\rho^{2}}}\right) .
\end{aligned}
$$

Hence, by the law of large numbers, $L_{n} / n$ converges in law to the random variable $p(Z)$ valued in $(0,1)$. In order that $\left\{L_{n} \geq l_{n}\right\}$ becomes a rare event (without being impossible) as $n$ increases, we therefore let $l_{n} / n$ approach 1 from below. We then set

$$
l_{n}=n q_{n}, \quad \text { with } q_{n}<1, q_{n} \nearrow 1 \text { as } n \rightarrow \infty .
$$

Actually, we assume that the rate of increase of $q_{n}$ to 1 is of order $n^{-a}$ with $a \leq 1$ :

$$
1-q_{n}=O\left(n^{-a}\right), \quad \text { with } 0<a \leq 1 .
$$

We then state the large deviations result for the large loss threshold regime.

Theorem 5.1 In the single-factor homogeneous portfolio credit risk model (5.1), and with large threshold $l_{n}$ as in (5.3)-(5.4), we have

$$
\lim _{n \rightarrow \infty} \frac{1}{\ln n} \ln \mathbb{P}\left[L_{n} \geq n q_{n}\right]=-a \frac{1-\rho^{2}}{\rho^{2}} .
$$

Observe that in the above theorem, we normalize by $\ln n$, indicating that the probability decays like $n^{-\gamma}$, with $\gamma=a\left(1-\rho^{2}\right) / \rho^{2}$. We find that the decay rate is determined by the effect of the dependence structure in the Gaussian copula model. When $\rho$ is small (weak dependence between sources of credit risk), large losses occur very rarely, which is formalized by a high decay rate. In the opposite case, this decay rate is small when $\rho$ tends to one, which means that large losses are most likely to result from systematic risk factors. 
Proof. 1) We first prove the lower bound :

$$
\liminf _{n \rightarrow \infty} \frac{1}{\ln n} \ln \mathbb{P}\left[L_{n} \geq n q_{n}\right] \geq-a \frac{1-\rho^{2}}{\rho^{2}} .
$$

From Bayes formula, we have

$$
\begin{aligned}
\mathbb{P}\left[L_{n} \geq n q_{n}\right] & \geq \mathbb{P}\left[L_{n} \geq n q_{n}, p(Z)=q_{n}\right] \\
& =\mathbb{P}\left[L_{n} \geq n q_{n} \mid p(Z) \geq q_{n}\right] \mathbb{P}\left[p(Z) \geq q_{n}\right] .
\end{aligned}
$$

For any $n \geq 1$, we define $z_{n} \in \mathbb{R}$ the solution to

$$
p\left(z_{n}\right)=q_{n}, \quad n \geq 1 .
$$

Since $p($.$) is an increasing one to one function, we have \left\{p(Z) \geq q_{n}\right\}=\left\{Z \geq z_{n}\right\}$. Moreover, observing that $L_{n}$ is an increasing function of $Z$, we get

$$
\begin{aligned}
\mathbb{P}\left[L_{n} \geq n q_{n} \mid p(Z) \geq q_{n}\right] & =\mathbb{P}\left[L_{n} \geq n q_{n} \mid Z \geq z_{n}\right] \\
& \geq \mathbb{P}\left[L_{n} \geq n q_{n} \mid Z=z_{n}\right]=\mathbb{P}\left[L_{n} \geq n q_{n} \mid p(Z)=q_{n}\right]
\end{aligned}
$$

so that from (5.6)

$$
\mathbb{P}\left[L_{n} \geq n q_{n}\right] \geq \mathbb{P}\left[L_{n} \geq n q_{n} \mid p(Z)=q_{n}\right] \mathbb{P}\left[Z \geq z_{n}\right] .
$$

Now given $p(Z)=q_{n}, L_{n}$ is binomially distributed with parameters $n$ and $q_{n}$, and thus

$$
\mathbb{P}\left[L_{n} \geq n q_{n} \mid p(Z)=q_{n}\right] \geq 1-\Phi(0)=\frac{1}{2}(>0) .
$$

We focus on the tail probability $\mathbb{P}\left[Z \geq z_{n}\right]$ as $n$ goes to infinity. First, observe that since $q_{n}$ goes to 1 , we have $z_{n}$ going to infinity as $n$ tends to infinity. Furthermore, from the expression (5.2) of $p(z)$, the rate of decrease (5.4), and using the property that $1-\Phi(x) \simeq$ $\varphi(x) / x$ as $x \rightarrow \infty$, we have

$$
\begin{aligned}
O\left(n^{-a}\right)=1-q_{n}=1-p\left(z_{n}\right) & =1-\Phi\left(\frac{\rho z_{n}+\Phi^{-1}(p)}{\sqrt{1-\rho^{2}}}\right) \\
& \simeq \frac{\sqrt{1-\rho^{2}}}{\rho z_{n}+\Phi^{-1}(p)} \exp \left(-\frac{1}{2}\left(\frac{\rho z_{n}+\Phi^{-1}(p)}{\sqrt{1-\rho^{2}}}\right)^{2}\right),
\end{aligned}
$$

as $n \rightarrow \infty$, so that by taking logarithm :

$$
a \ln n-\frac{1}{2} \frac{\rho^{2} z_{n}^{2}}{1-\rho^{2}}-\ln z_{n}=O(1)
$$

This implies

$$
\lim _{n \rightarrow \infty} \frac{z_{n}^{2}}{\ln n}=2 a \frac{1-\rho^{2}}{\rho^{2}}
$$

By writing

$$
\begin{aligned}
\mathbb{P}\left[Z \geq z_{n}\right] & =\mathbb{P}\left[z_{n} \leq Z \leq z_{n}+1\right] \\
& \geq \frac{1}{\sqrt{2 \pi}} \exp \left(-\frac{1}{2}\left(z_{n}+1\right)^{2}\right)
\end{aligned}
$$


we deduce with (5.9)

$$
\liminf _{n \rightarrow \infty} \frac{1}{\ln n} \ln \mathbb{P}\left[Z \geq z_{n}\right] \geq a \frac{1-\rho^{2}}{\rho^{2}} .
$$

Combining with (5.7) and (5.8), we get the required lower bound (5.5).

2) We now focus on the upper bound

$$
\limsup _{n \rightarrow \infty} \frac{1}{\ln n} \ln \mathbb{P}\left[L_{n} \geq n q_{n}\right] \leq-a \frac{1-\rho^{2}}{\rho^{2}} .
$$

We introduce the conditional c.g.f. of $Y_{k}$ :

$$
\begin{aligned}
\Gamma(\theta, z) & =\ln \mathbb{E}\left[e^{\theta Y_{k}} \mid Z=z\right] \\
& =\ln \left(1-p(z)+p(z) e^{\theta}\right) .
\end{aligned}
$$

Then, for any $\theta \geq 0$, we get by Chebichev's inequality,

$$
\mathbb{P}\left[L_{n} \geq n q_{n} \mid Z\right] \leq \mathbb{E}\left[e^{\theta\left(L_{n}-n q_{n}\right)} \mid Z\right]=e^{-n\left(\theta q_{n}-\Gamma(\theta, Z)\right)},
$$

so that

$$
\mathbb{P}\left[L_{n} \geq n q_{n} \mid Z\right] \leq e^{-n \Gamma^{*}\left(q_{n}, Z\right)}
$$

where

$$
\Gamma^{*}(q, z)=\sup _{\theta \geq 0}[\theta q-\Gamma(\theta, z)]=\left\{\begin{array}{cl}
0, & \text { if } q \leq p(z) \\
q \ln \left(\frac{q}{p(z)}\right)+(1-q) \ln \left(\frac{1-q}{1-p(z)}\right), & \text { if } p(z)<q \leq 1 .
\end{array}\right.
$$

By taking expectation on both sides on (5.13), we get

$$
\mathbb{P}\left[L_{n} \geq n q_{n}\right] \leq \mathbb{E}\left[e^{F_{n}(Z)}\right],
$$

where we set $F_{n}(z)=-n \Gamma^{*}\left(q_{n}, z\right)$. Since $\rho>0$, the function $p(z)$ is increasing in $z$, so $\Gamma(\theta, z)$ is an increasing function of $z$ for all $\theta \geq 0$. Hence, $F_{n}(z)$ is an increasing function of $z$, which is nonpositive and attains its maximum value 0 , for all $z$ s.t. $q_{n}=p\left(z_{n}\right) \leq p(z)$, i.e. $z \geq z_{n}$. Moreover, by differentiation, we can check that $F_{n}$ is a concave function of $z$. We now introduce a change of measure. The idea is to shift the factor mean to reduce the variance of the term $e^{F_{n}(Z)}$ in the r.h.s. of (5.14). We consider the change of measure $\mathbb{P}_{\mu}$ that puts the distribution of $Z$ to $\mathcal{N}(\mu, 1)$. Its likelihood ratio is given by

$$
\frac{d \mathbb{P}_{\mu}}{d \mathbb{P}}=\exp \left(\mu Z-\frac{1}{2} \mu^{2}\right),
$$

so that

$$
\mathbb{E}\left[e^{F_{n}(Z)}\right]=\mathbb{E}_{\mu}\left[e^{F_{n}(Z)-\mu Z+\frac{1}{2} \mu^{2}}\right]
$$

where $\mathbb{E}_{\mu}$ denotes the expectation under $\mathbb{P}_{\mu}$. By concavity of $F_{n}$, we have $F_{n}(Z) \leq F_{n}(\mu)+$ $F_{n}^{\prime}(\mu)(Z-\mu)$, so that

$$
\mathbb{E}\left[e^{F_{n}(Z)}\right] \leq \mathbb{E}_{\mu}\left[e^{F_{n}(\mu)+\left(F_{n}^{\prime}(\mu)-\mu\right) Z-\mu F_{n}^{\prime}(\mu)+\frac{1}{2} \mu^{2}}\right] .
$$


We now choose $\mu=\mu_{n}$ solution to

$$
F_{n}^{\prime}\left(\mu_{n}\right)=\mu_{n}
$$

so that the term in the expectation in the r.h.s. of (5.15) does not depend on $Z$, and is therefore a constant term (with zero-variance). Such a $\mu_{n}$ exists, since, by strict concavity of the function $z \rightarrow F_{n}(z)-\frac{1}{2} z^{2}$, equation (5.16) is the first-order equation associated to the optimization problem :

$$
\mu_{n}=\arg \max _{\mu \in \mathbb{R}}\left[F_{n}(\mu)-\frac{1}{2} \mu^{2}\right]
$$

With this choice of factor mean $\mu_{n}$, and by inequalities (5.14), (5.15), we get

$$
\mathbb{P}\left[L_{n} \geq n q_{n}\right] \leq e^{F_{n}\left(\mu_{n}\right)-\frac{1}{2} \mu_{n}^{2}} .
$$

We now prove that $\mu_{n} / z_{n}$ converges to 1 as $n$ goes to infinity. Actually, we show that for all $\varepsilon>0$, there is $n_{0}$ large enough so that for all $n \geq n_{0}, z_{n}(1-\varepsilon)<\mu_{n}<z_{n}$. Since $F_{n}^{\prime}\left(\mu_{n}\right)-\mu_{n}=0$, and the function $F_{n}^{\prime}(z)-z$ is decreasing by concavity $F_{n}(z)-z^{2} / 2$, it suffices to show that

$$
F_{n}^{\prime}\left(z_{n}(1-\varepsilon)\right)-z_{n}(1-\varepsilon)>0 \quad \text { and } \quad F_{n}^{\prime}\left(z_{n}\right)-z_{n}<0
$$

We have

$$
F_{n}^{\prime}(z)=n\left(\frac{p\left(z_{n}\right)}{p(z)}-\frac{1-p\left(z_{n}\right)}{1-p(z)}\right) \varphi\left(\frac{\rho z+\Phi^{-1}(p)}{\sqrt{1-\rho^{2}}}\right) \frac{\rho}{\sqrt{1-\rho^{2}}} .
$$

The second inequality in (5.18) holds since $F_{n}^{\prime}\left(z_{n}\right)=0$ and $z_{n}>0$ for $q_{n}>p$, hence for $n$ large enough. Actually, $z_{n}$ goes to infinity as $n$ goes to infinity from (5.9). For the first inequality in (5.18), we use the property that $1-\Phi(x) \simeq \varphi(x) / x$ as $x \rightarrow \infty$, so that

$$
\lim _{n \rightarrow \infty} \frac{p\left(z_{n}\right)}{p\left(z_{n}(1-\varepsilon)\right)}=1, \quad \text { and } \quad \lim _{n \rightarrow \infty} \frac{1-p\left(z_{n}\right)}{1-p\left(z_{n}(1-\varepsilon)\right)}=0 .
$$

From (5.9), we have

$$
\varphi\left(\frac{\rho z_{n}(1-\varepsilon)+\Phi^{-1}(p)}{\sqrt{1-\rho^{2}}}\right)=0\left(n^{-a(1-\varepsilon)^{2}}\right)
$$

and therefore

$$
F_{n}^{\prime}\left(z_{n}(1-\varepsilon)\right)=0\left(n^{1-a(1-\varepsilon)^{2}}\right)
$$

Moreover, from (5.9) and as $a \leq 1$, we have

$$
z_{n}(1-\varepsilon)=0(\sqrt{\ln n})=o\left(n^{1-a(1-\varepsilon)^{2}}\right)
$$

We deduce that for $n$ large enough $F_{n}^{\prime}\left(z_{n}(1-\varepsilon)\right)-z_{n}(1-\varepsilon)>0$ and so (5.18). 
Finally, recalling that $F_{n}$ is nonpositive, and from (5.17), we obtain :

$$
\limsup _{n \rightarrow \infty} \frac{1}{\ln n} \ln \mathbb{P}\left[L_{n} \geq n q_{n}\right] \leq-\frac{1}{2} \lim _{n \rightarrow} \frac{\mu_{n}^{2}}{\ln n}=-\frac{1}{2} \lim _{n \rightarrow} \frac{z_{n}^{2}}{\ln n}=-a \frac{1-\rho^{2}}{\rho^{2}} .
$$

\section{Application : asymptotic optimality of two-step importance sampling estimator}

Consider the estimation problem of $\mathbb{P}\left[L_{n} \geq n q\right]$. We apply a two-step importance sampling (IS) by using IS conditional on the common factors $Z$ and IS to the distribution of the factors $Z$. Observe that conditioning on $Z$ reduces to the problem of the independent case studied in the previous paragraph, with default probability $p(Z)$ as defined in (5.2), and c.g.f. $\Gamma(., Z)$ in (5.11). Choose $\theta_{q_{n}}(Z) \geq 0$ attaining the $\operatorname{argmax}$ in $\theta q_{n}-\Gamma(\theta, Z)$, and return the estimator

$$
\exp \left(-\theta_{q_{n}}(Z) L_{n}+n \Gamma\left(\theta_{q_{n}}(Z), Z\right)\right) 1_{L_{n} \geq n q_{n}},
$$

where $L_{n}$ is sampled with a default probability $p\left(\theta_{q}(Z), Z\right)=p(Z) e^{\theta_{q_{n}}(Z)} /(1-p(Z)+$ $\left.p(Z) e^{\theta_{q_{n}}(Z)}\right)$. This provides an unbiased conditional estimator of $\mathbb{P}\left[L_{n} \geq n q_{n} \mid Z\right]$ and an asymptotically optimal conditional variance. We further apply IS to the factor $Z \sim \mathcal{N}(0,1)$ under $\mathbb{P}$, by shifting the factor mean to $\mu$, and then considering the estimator

$$
\exp \left(-\mu Z+\frac{1}{2} \mu^{2}\right) \exp \left(-\theta_{q_{n}}(Z) L_{n}+n \Gamma\left(\theta_{q_{n}}(Z), Z\right)\right) 1_{L_{n} \geq n q_{n}}
$$

where $Z$ is sampled from $\mathcal{N}(\mu, 1)$. To summarize, the two-step IS estimator is generated as follows :

- Sample $Z$ from $\mathcal{N}(\mu, 1)$

- Compute $\theta_{q_{n}}(Z)$ and $p\left(\theta_{q_{n}}(Z), Z\right)$

- Return the estimator (5.20) where $L_{n}$ is sampled with default probability $p\left(\theta_{q_{n}}(Z), Z\right)$.

By construction, this provides an unbiaised estimator of $\mathbb{P}\left[L_{n} \geq n q_{n}\right]$, and the key point is to specify the choice of $\mu$ in order to reduce the global variance or equivalently the second moment $M_{n}^{2}\left(\mu, q_{n}\right)$ of this estimator. First, recall from Cauchy-Schwarz's inequality : $M_{n}^{2}\left(\mu, q_{n}\right) \geq\left(\mathbb{P}\left[L_{n} \geq n q\right]\right)^{2}$, so that the fastest possible rate of decay of $M_{n}^{2}\left(\mu, q_{n}\right)$ is twice the probability itself :

$$
\liminf _{n \rightarrow \infty} \frac{1}{\ln n} \ln M_{n}^{2}\left(q_{n}, \mu\right) \geq 2 \lim _{n \rightarrow \infty} \frac{1}{\ln n} \ln \mathbb{P}\left[L_{n} \geq n q_{n}\right] .
$$

To achieve this twice rate, we proceed as follows. Denoting by $\overline{\mathbb{E}}$ the expectation under the IS distribution, we have

$$
\begin{aligned}
M_{n}^{2}\left(\mu, q_{n}\right) & =\overline{\mathbb{E}}\left[\exp \left(-2 \mu Z+\mu^{2}\right) \exp \left(-2 \theta_{q_{n}}(Z) L_{n}+2 n \Gamma\left(\theta_{q_{n}}(Z), Z\right)\right) 1_{L_{n} \geq n q_{n}}\right] \\
& \leq \overline{\mathbb{E}}\left[\exp \left(-2 \mu Z+\mu^{2}\right) \exp \left(-2 n \theta_{q_{n}}(Z) q_{n}+2 n \Gamma\left(\theta_{q_{n}}(Z), Z\right)\right)\right] \\
& =\overline{\mathbb{E}}\left[\exp \left(-2 \mu Z+\mu^{2}+2 F_{n}(Z)\right)\right]
\end{aligned}
$$


by definition of $\theta_{q_{n}}(Z)$ and $F_{n}(z)=-n \sup _{\theta \geq 0}\left[\theta q_{n}-\Gamma(\theta, z)\right]$ introduced in the proof of the upper bound in Theorem 5.1. As in (5.15), (5.17), by choosing $\mu=\mu_{n}$ solution to $F_{n}^{\prime}\left(\mu_{n}\right)$ $=\mu_{n}$, we then get

$$
M_{n}^{2}\left(\mu_{n}, q_{n}\right) \leq \exp \left(2 F_{n}\left(\mu_{n}\right)-\mu_{n}^{2}\right) \leq \exp \left(-\mu_{n}^{2}\right)
$$

since $F_{n}$ is nonpositive. From (5.19), this yields

$$
\limsup _{n \rightarrow \infty} \frac{1}{\ln n} \ln M_{n}^{2}\left(\mu_{n}, q_{n}\right) \leq-2 a \frac{1-\rho^{2}}{\rho^{2}}=2 \lim _{n \rightarrow \infty} \frac{1}{\ln n} \ln \mathbb{P}\left[L_{n} \geq n q_{n}\right],
$$

which proves together with (5.21) that

$$
\lim _{n \rightarrow \infty} \frac{1}{\ln n} \ln M_{n}^{2}\left(\mu_{n}, q_{n}\right)=-2 a \frac{1-\rho^{2}}{\rho^{2}}=2 \lim _{n \rightarrow \infty} \frac{1}{\ln n} \ln \mathbb{P}\left[L_{n} \geq n q_{n}\right],
$$

and thus the estimator (5.20) for the choice $\mu=\mu_{n}$ is asymptotically optimal. The choice of $\mu=z_{n}$ also leads to an asymptotically optimal estimator.

Remark 5.2 We also prove by similar methods large deviation results for the loss distribution in the limiting regime where individual loss probabilities decrease toward zero, see [26] for the details. This setting is relevant to portfolios of highly-rated obligors, for which one-year default probabilities are extremely small. This is also relevant to measuring risk over short time horizons. In this limiting regime, we set

$$
l_{n}=n q, \quad \text { with } 0<q<1, \quad p=p_{n}=O\left(e^{-n a}\right) \text {, with } a>0 .
$$

Then,

$$
\lim _{n \rightarrow \infty} \frac{1}{n} \ln \mathbb{P}\left[L_{n} \geq n q\right]=-\frac{a}{\rho^{2}},
$$

and we may construct similarly as in the case of large losses, a two-step IS asymptotically optimal estimator.

\subsection{A large deviations approach to optimal long term investment}

\subsubsection{An asymptotic outperforming benchmark criterion}

A popular approach for institutional managers is concerned about the performance of their portfolio relative to the achievement of a given benchmark. This means that investors are interested in maximizing the probability that their wealth exceed a predetermined index. Equivalently, this may be also formulated as the problem of minimizing the probability that the wealth of the investor falls below a specified value. This target problem was studied by several authors for a goal achievement in finite time horizon, see e.g. [11] or [20]. Recently, and in a static framework, the paper [40] considered an asymptotic version of this outperformance criterion when time horizon goes to infinity, which leads to a large deviations portfolio criterion. To illustrate the purpose, let us consider the following toy example. Suppose that an investor trades a number $\alpha$ of shares in stock of price $S$, and 
keep it until time $T$. Her wealth at time $T$ is then $X_{T}^{\alpha}=\alpha S_{T}$. For simplicity, we take a Bachelier model for the stock price : $S_{t}=\mu t+\sigma W_{t}$, where $W$ is a brownian motion. We now look at the behavior of the average wealth when time horizon $T$ goes to infinity. By the law of large numbers, for any $\alpha \in \mathbb{R}$, the average wealth converges a.s. to :

$$
\bar{X}_{T}^{\alpha}:=\frac{X_{T}^{\alpha}}{T}=\alpha \mu+\alpha \sigma \frac{W_{T}}{T} \longrightarrow \alpha \mu,
$$

when $T$ goes to infinity. When considering positive stock price, as in the Black-Scholes model, the relevant ergodic mean is the average of the growth rate, i.e. the logarithm of the wealth. Fix some benchmark level $x \in \mathbb{R}$. Then, from Cramer's theorem, the probability of outperforming $x$ decays exponentially fast as :

$$
\mathbb{P}\left[\bar{X}_{T}^{\alpha} \geq x\right] \simeq e^{-I(x, \alpha) T},
$$

in the sense that $\lim _{T \rightarrow \infty} \frac{1}{T} \ln \mathbb{P}\left[\bar{X}_{T}^{\alpha} \geq x\right]=-I(x, \alpha)$, where

$$
\begin{aligned}
I(x, \alpha) & =\sup _{\theta \in \mathbb{R}}[\theta x-\Gamma(\theta, \alpha)] \\
\Gamma(\theta, \alpha) & =\frac{1}{T} \ln \mathbb{E}\left[e^{\theta X_{T}^{\alpha}}\right] .
\end{aligned}
$$

Thus, the lower is the decay rate $I(x, \alpha)$, the more chance there is of realizing a portfolio performance above $x$. The asymptotic version of the outperforming benchmark criterion is then formulated as :

$$
\sup _{\alpha \in \mathbb{R}} \lim _{T \rightarrow \infty} \frac{1}{T} \ln \mathbb{P}\left[\bar{X}_{T}^{\alpha} \geq x\right]=-\inf _{\alpha \in \mathbb{R}} I(x, \alpha) .
$$

In this simple example, the quantities involved are all explicit :

$$
\begin{aligned}
& \Gamma(\theta, \alpha)=\theta \alpha \mu+\frac{(\theta \alpha \sigma)^{2}}{2} \\
& I(x, \alpha)=\left\{\begin{array}{cl}
\frac{1}{2}\left(\frac{\alpha \mu-x}{\alpha \sigma}\right)^{2}, & \alpha \neq 0 \\
0, & \alpha=0, x=0 \\
\infty, & \alpha=0, x \neq 0 .
\end{array}\right.
\end{aligned}
$$

The solution to (5.22) is then given by $\alpha^{*}=x / \mu$, which means that the associated expected wealth $\mathbb{E}\left[\bar{X}_{T}^{\alpha^{*}}\right]$ is equal to the target $x$.

We now develop an asymptotic dynamic version of the outperformance management criterion due to [36. Such a problem corresponds to an ergodic objective of beating a given benchmark, and may be of particular interest for institutional managers with long term horizon, like mutual funds. On the other hand, stationary long term horizon problems are expected to be more tractable than finite horizon problems, and should provide some good insight for management problems with long, but finite, time horizon.

We formulate the problem in a rather abstract setting. Let $Z=(X, Y)$ be a process valued in $\mathbb{R} \times \mathbb{R}^{d}$, controlled by $\alpha$, a control process valued in some subset $A$ of $\mathbb{R}^{q}$. We denote by $\mathcal{A}$ the set of control processes. As usual, to alleviate notations, we omitted the 
dependence of $Z=(X, Y)$ in $\alpha \in \mathcal{A}$. We shall then study the large deviations control problem :

$$
v(x)=\sup _{\alpha \in \mathcal{A}} \limsup _{T \rightarrow \infty} \frac{1}{T} \ln \mathbb{P}\left[\bar{X}_{T} \geq x\right], \quad x \in \mathbb{R},
$$

where $\bar{X}_{T}=X_{T} / T$. The variable $X$ should typically be viewed in finance as the (logarithm) of the wealth process, $Y$ are factors on market (stock, volatility ...), and $\alpha$ represents the trading portfolio.

\subsubsection{Duality to the large deviations control problem}

The large deviations control problem (5.23) is a non standard stochastic control problem, where the objective is usually formulated as an expectation of some functional to optimize. In particular, in a Markovian continuous-time setting, we do not know if there is a dynamic programming principle and a corresponding Hamilton-Jacobi-Bellman equation for our problem. We shall actually adopt a duality approach based on the relation relating rate function of a LDP and cumulant generating function. The formal derivation is the following. Given $\alpha \in \mathcal{A}$, if there is a $\operatorname{LDP}$ for $\bar{X}_{T}=X_{T} / T$, its rate function $I(., \alpha)$ should be related by the Fenchel-Legendre transform :

$$
I(x, \alpha)=\sup _{\theta}[\theta x-\Gamma(\theta, \alpha)]
$$

to the c.g.f.

$$
\Gamma(\theta, \alpha)=\limsup _{T \rightarrow \infty} \frac{1}{T} \ln \mathbb{E}\left[e^{\theta X_{T}}\right]
$$

In this case, we would get

$$
\begin{aligned}
v(x)=\sup _{\alpha \in \mathcal{A}} \limsup _{T \rightarrow \infty} \frac{1}{T} \ln \mathbb{P}\left[\bar{X}_{T} \geq x\right] & =-\inf _{\alpha \in \mathcal{A}} I(x, \alpha) \\
& =-\inf _{\alpha \in \mathcal{A}} \sup _{\theta}[\theta x-\Gamma(\theta, \alpha)],
\end{aligned}
$$

and so, provided that one could intervert infinum and supremum in the above relation (actually, the minmax theorem does not apply since $\mathcal{A}$ is not necessarily compact and $\alpha \rightarrow$ $\theta x-\Gamma(\theta, \alpha)$ is not convex) :

$$
v(x)=-\sup _{\theta}[\theta x-\Gamma(\theta)]
$$

where

$$
\Gamma(\theta)=\sup _{\alpha \in \mathcal{A}} \Gamma(\theta, \alpha)=\sup _{\alpha \in \mathcal{A}} \limsup _{T \rightarrow \infty} \frac{1}{T} \ln \mathbb{E}\left[e^{\theta X_{T}}\right] .
$$

Problem (5.26) is the dual problem via (5.25) to the original problem (5.23). We shall see in the next section that $(5.26)$ can be reformulated as a risk-sensitive ergodic control problem, which is more tractable than (5.23) and is studied by dynamic programming methods leading in some cases to explicit calculations. 
First, we show rigorously the duality relation between the large deviations control problem and the risk-sensitive control problem and how the optimal controls to the former one are related to the latter one. This result may be viewed as an extension of the Gärtner-Ellis theorem with control components.

Theorem 5.2 Suppose that there exists $\bar{\theta} \in(0, \infty]$ such that for all $\theta \in[0, \bar{\theta})$, there exists a solution $\hat{\alpha}(\theta) \in \mathcal{A}$ to the dual problem $\Gamma(\theta)$, with a limit in (5.24), i.e.

$$
\Gamma(\theta)=\lim _{T \rightarrow \infty} \frac{1}{T} \ln \mathbb{E}\left[\exp \left(\theta X_{T}^{\hat{\alpha}(\theta)}\right)\right]
$$

Suppose also that $\Gamma(\theta)$ is continuously differentiable on $[0, \bar{\theta})$. Then for all $x<\Gamma^{\prime}(\bar{\theta}):=$ $\lim _{\lambda / \bar{\theta}} \Gamma^{\prime}(\theta)$, we get

$$
v(x)=-\sup _{\theta \in[0, \bar{\theta})}[\theta x-\Gamma(\theta)] .
$$

Moreover, the sequence of controls

$$
\alpha_{t}^{*, n}=\left\{\begin{array}{rlrl}
\hat{\alpha}_{t}\left(\theta\left(x+\frac{1}{n}\right)\right), & \Gamma^{\prime}(0) & <x<\Gamma^{\prime}(\bar{\theta}) \\
\hat{\alpha}_{t}\left(\theta\left(\Gamma^{\prime}(0)+\frac{1}{n}\right)\right), & & x & \leq \Gamma^{\prime}(0),
\end{array}\right.
$$

with $\theta(x) \in(0, \bar{\theta})$ s.t. $\Gamma^{\prime}(\theta(x))=x \in\left(\Gamma^{\prime}(0), \Gamma^{\prime}(\bar{\theta})\right)$, is nearly optimal in the sense that

$$
\lim _{n \rightarrow \infty} \limsup _{T \rightarrow \infty} \frac{1}{T} \ln \mathbb{P}\left[\bar{X}_{T}^{\alpha^{*, n}} \geq x\right]=v(x) .
$$

\section{Proof.}

Step 1. Let us consider the Fenchel-Legendre transform of the convex function $\Gamma$ on $[0, \bar{\theta})$ :

$$
\Gamma^{*}(x)=\sup _{\theta \in[0, \bar{\theta})}[\theta x-\Gamma(\theta)], \quad x \in \mathbb{R} .
$$

Since $\Gamma$ is $C^{1}$ on $\left[0, \bar{\theta}\right.$ ), it is well-known (see e.g. Lemma 2.3.9 in [13]) that the function $\Gamma^{*}$ is convex, nondecreasing and satisfies :

$$
\begin{gathered}
\Gamma^{*}(x)=\left\{\begin{array}{cc}
\theta(x) x-\Gamma(\theta(x)), & \text { if } \Gamma^{\prime}(0)<x<\Gamma^{\prime}(\bar{\theta}) \\
0, & \text { if } x \leq \Gamma^{\prime}(0),
\end{array}\right. \\
\theta(x) x-\Gamma^{*}(x)>\theta(x) x^{\prime}-\Gamma^{*}\left(x^{\prime}\right), \quad \forall \Gamma^{\prime}(0)<x<\Gamma^{\prime}(\bar{\theta}), \forall x^{\prime} \neq x,
\end{gathered}
$$

where $\theta(x) \in(0, \bar{\theta})$ is s.t. $\Gamma^{\prime}(\theta(x))=x \in\left(\Gamma^{\prime}(0), \Gamma^{\prime}(\bar{\theta})\right)$. Moreover, $\Gamma^{*}$ is continuous on $\left(-\infty, \Gamma^{\prime}(\bar{\theta})\right)$.

Step 2: Upper bound. For all $x \in \mathbb{R}, \alpha \in \mathcal{A}$, an application of Chebycheff's inequality yields :

$$
\mathbb{P}\left[\bar{X}_{T} \geq x\right] \leq \exp (-\theta x T) \mathbb{E}\left[\exp \left(\theta X_{T}\right)\right], \quad \forall \theta \in[0, \bar{\theta})
$$


and so

$$
\limsup _{T \rightarrow \infty} \frac{1}{T} \ln \mathbb{P}\left[\bar{X}_{T} \geq x\right] \leq-\theta x+\limsup _{T \rightarrow \infty} \frac{1}{T} \ln \mathbb{E}\left[\exp \left(\theta X_{T}\right)\right], \quad \forall \theta \in[0, \bar{\theta}) .
$$

By definitions of $\Gamma$ and $\Gamma^{*}$, we deduce :

$$
\sup _{\alpha \in \mathcal{A}} \limsup _{T \rightarrow \infty} \frac{1}{T} \ln \mathbb{P}\left[\bar{X}_{T}^{\alpha} \geq x\right] \leq-\Gamma^{*}(x) .
$$

Step 3 : Lower bound. Given $x<\Gamma^{\prime}(\bar{\theta})$, let us define the probability measure $\mathbb{Q}_{T}^{n}$ on $\left(\Omega, \mathcal{F}_{T}\right)$ via :

$$
\frac{d \mathbb{Q}_{T}^{n}}{d \mathbb{P}}=\exp \left[\theta\left(x_{n}\right) X_{T}^{\alpha^{*, n}}-\Gamma_{T}\left(\theta\left(x_{n}\right), \alpha^{*, n}\right)\right],
$$

where $x_{n}=x+1 / n$ if $x>\Gamma^{\prime}(0), x_{n}=\Gamma^{\prime}(0)+1 / n$ otherwise, $\alpha^{*, n}=\hat{\alpha}\left(\theta\left(x_{n}\right)\right)$, and

$$
\Gamma_{T}(\theta, \alpha)=\ln \mathbb{E}\left[\exp \left(\theta X_{T}^{\alpha}\right)\right], \quad \theta \in[0, \bar{\theta}), \alpha \in \mathcal{A} .
$$

Here $n$ is large enough so that $x+1 / n<\Gamma^{\prime}(\bar{\theta})$. We now take $\varepsilon>0$ small enough so that $x \leq x_{n}-\varepsilon$ and $x_{n}+\varepsilon<\Gamma^{\prime}(\bar{\theta})$. We then have :

$$
\begin{aligned}
\frac{1}{T} \ln \mathbb{P}\left[\bar{X}_{T}^{\left.\alpha^{*, n} \geq x\right] \geq}\right. & \frac{1}{T} \ln \mathbb{P}\left[x_{n}-\varepsilon<\bar{X}_{T}^{\alpha^{*, n}}<x_{n}+\varepsilon\right] \\
= & \frac{1}{T} \ln \left(\int \frac{d \mathbb{P}}{d \mathbb{Q}_{T}^{n}} 1_{\left\{x_{n}-\varepsilon<\bar{X}_{T}^{\alpha^{*, n}}<x_{n}+\varepsilon\right\}} d \mathbb{Q}_{T}^{n}\right) \\
\geq & -\theta\left(x_{n}\right)\left(x_{n}+\varepsilon\right)+\frac{1}{T} \Gamma_{T}\left(\theta\left(x_{n}\right), \alpha^{*, n}\right) \\
& \quad+\frac{1}{T} \ln \mathbb{Q}_{T}^{n}\left[x_{n}-\varepsilon<\bar{X}_{T}^{\alpha^{*, n}}<x_{n}+\varepsilon\right],
\end{aligned}
$$

where we use (5.33) in the last inequality. By definition of the dual problem, this yields :

$$
\begin{aligned}
\liminf _{T \rightarrow \infty} \frac{1}{T} \ln \mathbb{P}\left[\bar{X}_{T}^{\alpha^{*, n}} \geq x\right] \geq- & \theta\left(x_{n}\right)\left(x_{n}+\varepsilon\right)+\Gamma\left(\theta\left(x_{n}\right)\right) \\
& +\liminf _{T \rightarrow \infty} \frac{1}{T} \ln \mathbb{Q}_{T}^{n}\left[x_{n}-\varepsilon<\bar{X}_{T}^{\alpha^{*, n}}<x_{n}+\varepsilon\right] \\
\geq \quad- & \Gamma^{*}\left(x_{n}\right)-\theta\left(x_{n}\right) \varepsilon \\
& +\liminf _{T \rightarrow \infty} \frac{1}{T} \ln \mathbb{Q}_{T}^{n}\left[x_{n}-\varepsilon<\bar{X}_{T}^{\alpha^{*, n}}<x_{n}+\varepsilon\right]
\end{aligned}
$$

where the second inequality follows by the definition of $\Gamma^{*}$ (and actually holds with equality due to $(5.30)$ ). We now show that :

$$
\liminf _{T \rightarrow \infty} \frac{1}{T} \ln Q_{T}^{n}\left[x_{n}-\varepsilon<\bar{X}_{T}^{\alpha^{*, n}}<x_{n}+\varepsilon\right]=0 .
$$

Denote by $\tilde{\Gamma}_{T}^{n}$ the c.g.f. under $Q_{T}^{n}$ of $X_{T}^{\alpha^{*, n}}$. For all $\zeta \in \mathbb{R}$, we have by (5.33) :

$$
\begin{aligned}
\tilde{\Gamma}_{T}^{n}(\zeta) & :=\ln E^{\mathbb{Q}_{T}^{n}}\left[\exp \left(\zeta X_{T}^{\alpha^{*, n}}\right)\right] \\
& =\Gamma_{T}\left(\theta\left(x_{n}\right)+\zeta, \alpha^{*, n}\right)-\Gamma_{T}\left(\theta\left(x_{n}\right), \alpha^{*, n}\right) .
\end{aligned}
$$


Therefore, by definition of the dual problem and (5.27), we have for all $\zeta \in\left[-\theta\left(x_{n}\right), \bar{\theta}-\right.$ $\left.\theta\left(x_{n}\right)\right)$ :

$$
\limsup _{T \rightarrow \infty} \frac{1}{T} \tilde{\Gamma}_{T}^{n}(\zeta) \leq \Gamma\left(\theta\left(x_{n}\right)+\zeta\right)-\Gamma\left(\theta\left(x_{n}\right)\right) .
$$

As in part 1) of this proof, by Chebycheff's inequality, we have for all $\zeta \in\left[0, \bar{\theta}-\theta\left(x_{n}\right)\right)$ :

$$
\begin{aligned}
\limsup _{T \rightarrow \infty} \frac{1}{T} \ln \mathbb{Q}_{T}^{n}\left[\bar{X}_{T}^{\alpha^{*, n}} \geq x_{n}+\varepsilon\right] & \leq-\zeta\left(x_{n}+\varepsilon\right)+\limsup _{T \rightarrow \infty} \frac{1}{T} \tilde{\Gamma}_{T}^{n}(\zeta) \\
& \leq-\zeta\left(x_{n}+\varepsilon\right)+\Gamma\left(\zeta+\theta\left(x_{n}\right)\right)-\Gamma\left(\theta\left(x_{n}\right)\right),
\end{aligned}
$$

where the second inequality follows from (5.36). We deduce

$$
\begin{aligned}
\limsup _{T \rightarrow \infty} \frac{1}{T} \ln \mathbb{Q}_{T}^{n}\left[\bar{X}_{T}^{\alpha^{*, n}} \geq x_{n}+\varepsilon\right] \leq & -\sup \left\{\zeta\left(x_{n}+\varepsilon\right)-\Gamma(\zeta): \zeta \in\left[\theta\left(x_{n}\right), \bar{\theta}\right)\right\} \\
& -\Gamma\left(\theta\left(x_{n}\right)\right)+\theta\left(x_{n}\right)\left(x_{n}+\varepsilon\right) \\
\leq & -\Gamma^{*}\left(x_{n}+\varepsilon\right)-\Gamma\left(\theta\left(x_{n}\right)\right)+\theta\left(x_{n}\right)\left(x_{n}+\varepsilon\right), \\
= & -\Gamma^{*}\left(x_{n}+\varepsilon\right)+\Gamma^{*}\left(x_{n}\right)+\varepsilon \theta\left(x_{n}\right),
\end{aligned}
$$

where the second inequality and the last equality follow from (5.30). Similarly, we have for all $\zeta \in\left[-\theta\left(x_{n}\right), 0\right]$ :

$$
\begin{aligned}
\limsup _{T \rightarrow \infty} \frac{1}{T} \ln \mathbb{Q}_{T}^{n}\left[\bar{X}_{T}^{\alpha^{*, n}} \leq x_{n}-\varepsilon\right] & \leq-\zeta\left(x_{n}-\varepsilon\right)+\limsup _{T \rightarrow \infty} \frac{1}{T} \tilde{\Gamma}_{T}^{n}(\zeta) \\
& \leq-\zeta\left(x_{n}-\varepsilon\right)+\Gamma\left(\theta\left(x_{n}\right)+\zeta\right)-\Gamma\left(\theta\left(x_{n}\right)\right),
\end{aligned}
$$

and so :

$$
\begin{aligned}
\limsup _{T \rightarrow \infty} \frac{1}{T} \ln \mathbb{Q}_{T}^{n}\left[\bar{X}_{T}^{\alpha^{*, n}} \leq x_{n}-\varepsilon\right] \leq & -\sup \left\{\zeta\left(x_{n}-\varepsilon\right)-\Gamma(\zeta): \zeta \in\left[0, \theta\left(x_{n}\right)\right]\right\} \\
& -\Gamma\left(\theta\left(x_{n}\right)\right)+\theta\left(x_{n}\right)\left(x_{n}-\varepsilon\right) \\
\leq & -\Gamma^{*}\left(x_{n}-\varepsilon\right)+\Gamma^{*}\left(\theta\left(x_{n}\right)\right)-\varepsilon \theta\left(x_{n}\right) .
\end{aligned}
$$

By (5.37)-(5.38), we then get :

$$
\begin{aligned}
& \limsup _{T \rightarrow \infty} \frac{1}{T} \ln \mathbb{Q}_{T}^{n}\left[\left\{\bar{X}_{T}^{\alpha^{*, n}} \leq x_{n}-\varepsilon\right\} \cup\left\{\bar{X}_{T}^{\alpha^{*, n}} \geq x_{n}+\varepsilon\right\}\right] \\
\leq & \max \left\{\limsup _{T \rightarrow \infty} \frac{1}{T} \ln \mathbb{Q}_{T}^{n}\left[\bar{X}_{T}^{\alpha^{*, n}} \geq x_{n}+\varepsilon\right] ; \limsup _{T \rightarrow \infty} \frac{1}{T} \ln \mathbb{Q}_{T}^{n}\left[\bar{X}_{T}^{\alpha^{*, n}} \leq x_{n}-\varepsilon\right]\right\} \\
\leq & \max \left\{-\Gamma^{*}\left(x_{n}+\varepsilon\right)+\Gamma^{*}\left(x_{n}\right)+\varepsilon \theta\left(x_{n}\right) ;-\Gamma^{*}\left(x_{n}-\varepsilon\right)+\Gamma^{*}\left(\theta\left(x_{n}\right)\right)-\varepsilon \theta\left(x_{n}\right)\right\} \\
< & 0,
\end{aligned}
$$

where the strict inequality follows from (5.31). This implies that $\mathbb{Q}_{T}^{n}\left[\left\{\bar{X}_{T}^{\alpha^{*, n}} \leq x_{n}-\varepsilon\right\} \cup\right.$ $\left.\left\{\bar{X}_{T}^{\alpha^{*, n}} \geq x_{n}+\varepsilon\right\}\right] \rightarrow 0$ and hence $\mathbb{Q}_{T}^{n}\left[x_{n}-\varepsilon<\bar{X}_{T}^{\alpha^{*, n}}<x_{n}+\varepsilon\right] \rightarrow 1$ as $T$ goes to infinity. In particular (5.35) is satisfied, and by sending $\varepsilon$ to zero in (5.34), we get :

$$
\liminf _{T \rightarrow \infty} \frac{1}{T} \ln \mathbb{P}\left[\bar{X}_{T}^{\alpha^{*, n}} \geq x\right] \geq-\Gamma^{*}\left(x_{n}\right) .
$$


By continuity of $\Gamma^{*}$ on $\left(-\infty, \Gamma^{\prime}(\bar{\theta})\right)$, we obtain by sending $n$ to infinity and recalling that $\Gamma^{*}(x)=0=\Gamma^{*}\left(\Gamma^{\prime}(0)\right)$ for $x \leq \Gamma^{\prime}(0)$ :

$$
\liminf _{n \rightarrow \infty} \liminf _{T \rightarrow \infty} \frac{1}{T} \ln \mathbb{P}\left[\bar{X}_{T}^{\alpha^{*, n}} \geq x\right] \geq-\Gamma^{*}(x) .
$$

This last inequality combined with (5.32) ends the proof.

Remark 5.3 Notice that in Theorem 5.2, the duality relation (5.28) holds for $x<\Gamma^{\prime}(\bar{\theta})$. When $\Gamma^{\prime}(\bar{\theta})=\infty$, we say that fonction $\Gamma$ is steep, so that (5.28) holds for all $x \in \mathbb{R}$. We illustrate in the next section different cases where $\Gamma$ is steep or not.

Remark 5.4 In financial applications, $X_{t}$ is the logarithm of an investor's wealth $V_{t}^{\alpha}$ at time $t, \alpha_{t}$ is the proportion of wealth invested in $q$ risky assets $S$ and $Y$ is some economic factor influencing the dynamics of $S$ and the savings account $S^{0}$. Hence, in a diffusion model, we have

$$
d X_{t}=\left[r\left(Y_{t}\right)+\alpha_{t}^{\prime}\left(\mu\left(Y_{t}\right)-r\left(Y_{t}\right) e_{q}\right)-\frac{1}{2}\left|\alpha_{t}^{\prime} \vartheta\left(Y_{t}\right)\right|^{2}\right] d t+\alpha_{t}^{\prime} \vartheta\left(Y_{t}\right) d W_{t},
$$

where $\mu(y)$ (resp. $\vartheta(y)$ ) is the rate of return (resp. volatility) of the risky assets, $r(y)$ is the interest rate, and $e_{q}$ is the unit vector in $\mathbb{R}^{q}$.

Notice that the value function of the dual problem can be written as :

$$
\Gamma(\theta)=\lim _{T \rightarrow \infty} \frac{1}{T} \ln E\left[U_{\theta}\left(V_{T}^{\hat{\alpha}(\theta)}\right)\right],
$$

where $U_{\theta}(c)=c^{\theta}$ is a power utility function with Constant Relative Risk Aversion (CRRA) $1-\theta>0$ provided that $\theta<1$. Then, Theorem 5.2 means that for any target level $x$, the optimal overperformance probability of growth rate is (approximately) directly related, for large $T$, to the expected CRRA utility of wealth, by :

$$
P\left[\bar{X}_{T}^{\alpha^{*}} \geq x\right] \approx E\left[U_{\theta(x)}\left(V_{T}^{\alpha^{*}}\right)\right] e^{-\theta(x) x T},
$$

with the convention that $\theta(x)=0$ for $x \leq \Gamma^{\prime}(0)$. Hence, $1-\theta(x)$ can be interpreted as a constant degree of relative risk aversion for an investor who has an overperformance target level $x$. Moreover, by strict convexity of function $\Gamma^{*}$ in (5.29), it is clear that $\theta(x)$ is strictly increasing for $x>\Gamma^{\prime}(0)$. So an investor with a higher target level $x$ has a lower degree of relative risk aversion $1-\theta(x)$. In summary, Theorem 5.2 (or relation (5.39)) inversely relates the target level of growth rate to the degree of relative risk aversion in expected utility theory.

\subsubsection{Explicit calculations to the dual risk-sensitive control problem}

We now show that the dual control problem $(5.26)$ may be transformed via a change of probability measure into a risk-sensitive control problem. We consider the framework of a general diffusion model for $Z=(X, Y)$ :

$$
\begin{aligned}
& d X_{t}=b\left(X_{t}, Y_{t}, \alpha_{t}\right) d t+\sigma\left(X_{t}, Y_{t}, \alpha_{t}\right) d W_{t} \text { in } \mathbb{R} \\
& d Y_{t}=\eta\left(X_{t}, Y_{t}, \alpha_{t}\right) d t+\sigma\left(X_{t}, Y_{t}, \alpha_{t}\right) d W_{t} \text { in } \mathbb{R}^{d},
\end{aligned}
$$


where $W$ is a $m$-dimensional brownian motion on a filtered probability space $(\Omega, \mathcal{F}, \mathbb{F}=$ $\left.\left(\mathcal{F}_{t}\right)_{t \geq 0}, \mathbb{P}\right)$, and $\alpha=\left(\alpha_{t}\right)_{t \geq 0}$, the control process, is $\mathbb{F}$-adapted and valued in some subset $A$ of $\mathbb{R}^{q}$. We denote $\mathcal{A}$ the set of control processes. The coefficients $b, \eta, \sigma$ and $\gamma$ are measurable functions of their arguments, and given $\alpha \in \mathcal{A}$ and an initial condition, we assume the existence and uniqueness of a strong solution to (5.40)-(5.41), which we also write by setting $Z=(X, Y)$ :

$$
d Z_{t}=B\left(Z_{t}, \alpha_{t}\right) d t+\Sigma\left(Z_{t}, \alpha_{t}\right) d W_{t} .
$$

From the dynamics of $X$ in (5.40), we may rewrite the Laplace transform of $X_{T}$ as :

$$
\begin{aligned}
\mathbb{E}\left[\exp \left(\theta X_{T}\right)\right] & =e^{\theta X_{0}} \mathbb{E}\left[\exp \left(\theta \int_{0}^{T} b\left(Z_{t}, \alpha_{t}\right) d t+\theta \int_{0}^{T} \sigma\left(Z_{t}, \alpha_{t}\right) d W_{t}\right)\right] \\
& =e^{\theta X_{0}} \mathbb{E}\left[\xi_{T}^{\alpha}(\theta) \exp \left(\int_{0}^{T} \ell\left(\theta, Z_{t}, \alpha_{t}\right) d t\right)\right],
\end{aligned}
$$

where

$$
\ell(\theta, z, a)=\theta b(z, a)+\frac{\theta^{2}}{2}|\sigma(z, a)|^{2},
$$

and $\xi_{t}^{\alpha}(\theta)$ is the Doléans-Dade exponential local martingale

$$
\begin{aligned}
\xi_{t}^{\alpha}(\theta) & =\mathcal{E}\left(\theta \int \sigma\left(Z_{u}, \alpha_{u}\right) d W_{u}\right)_{t} \\
& :=\exp \left(\theta \int_{0}^{t} \sigma\left(Z_{u}, \alpha_{u}\right) d W_{u}-\frac{\theta^{2}}{2} \int_{0}^{t}\left|\sigma\left(Z_{u}, \alpha_{u}\right)\right|^{2} d u\right), t \geq 0 .
\end{aligned}
$$

If $\xi^{\alpha}(\theta)$ is a "true" martingale, it defines a probability measure $\mathbb{Q}$ under which, by Girsanov's theorem, the dynamics of $Z$ is given by :

$$
d Z_{t}=G\left(\theta, Z_{t}, \alpha_{t}\right) d t+\Sigma\left(Z_{t}, \alpha_{t}\right) d W_{t}^{\mathbb{Q}},
$$

where $W^{\mathbb{Q}}$ is a $\mathbb{Q}$-Brownian motion and

$$
G(\theta, z, a)=\left(\begin{array}{c}
b(z, a)+\theta|\sigma(z, a)|^{2} \\
\eta(z, a)+\theta \gamma \sigma^{\prime}(z, a)
\end{array}\right) .
$$

Hence, the dual problem may be written as a stochastic control problem with exponential integral cost criterion :

$$
\Gamma(\theta)=\sup _{\alpha \in \mathcal{A}} \limsup _{T \rightarrow \infty} \frac{1}{T} \ln \mathbb{E}^{\mathbb{Q}}\left[\exp \left(\int_{0}^{T} \ell\left(\theta, Z_{t}, \alpha_{t}\right) d t\right)\right], \quad \theta \geq 0 .
$$

For fixed $\theta$, this is an ergodic risk-sensitive control problem which has been studied by several authors, see e.g. [18], [10] or [39] in a discrete-time setting. It admits a dynamic programming equation :

$$
\begin{aligned}
\Lambda(\theta)=\sup _{a \in A}[ & \frac{1}{2} \operatorname{tr}\left(\Sigma \Sigma^{\prime}(z, a) D^{2} \phi_{\theta}\right)+G(\theta, z, a) \cdot \nabla \phi_{\theta} \\
& \left.+\frac{1}{2}\left|\Sigma^{\prime}(z, a) \nabla \phi_{\theta}\right|^{2}+\ell(\theta, z, a)\right], \quad z \in \mathbb{R}^{d+1} .
\end{aligned}
$$


The unknown is the pair $\left(\Lambda(\theta), \phi_{\theta}\right) \in \mathbb{R} \times C^{2}\left(\mathbb{R}^{d+1}\right)$, and $\Lambda(\theta)$ is a candidate for $\Gamma(\theta)$. The above P.D.E. is formally derived by considering the finite horizon problem

$$
u_{\theta}(T, z)=\sup _{\alpha \in \mathcal{A}} \mathbb{E}^{\mathbb{Q}}\left[\exp \left(\int_{0}^{T} \ell\left(\theta, Z_{t}, \alpha_{t}\right) d t\right)\right],
$$

by writing the Bellman equation for this classical control problem and by making the logarithm transformation

$$
\ln u_{\theta}(T, z) \simeq \Lambda(\theta) T+\phi_{\theta}(z)
$$

for large $T$.

One can prove rigorously that a pair solution $\left(\Lambda(\theta), \phi_{\theta}\right)$ to the PDE (5.46) provides a solution $\Lambda(\theta)=\Gamma(\theta)$ to the dual problem (5.26), with an optimal control given by the argument max in (5.46). This is called a verification theorem in stochastic control theory. Actually, there may have multiple solutions $\phi_{\theta}$ to (5.46) (even up to a constant), and we need some ergodicity condition to select the good one that satisfies the verification theorem. We refer to [37] for the details, and we illustrate our purpose with an example with explicit calculations.

We consider a one-factor model where the bond price $S^{0}$ and the stock price $S$ evolve according to :

$$
\frac{d S_{t}^{0}}{S_{t}^{0}}=\left(a_{0}+b_{0} Y_{t}\right) d t, \quad \frac{d S_{t}}{S_{t}}=\left(a+b Y_{t}\right) d t+\sigma d W_{t},
$$

with a factor $Y$ as an Ornstein-Uhlenbeck ergodic process:

$$
d Y_{t}=-k Y_{t} d t+d B_{t}
$$

where $a_{0}, b_{0}, a, b$ are constants, $k, \sigma$ are positive constants, and $W, B$ are two brownian motions, supposed non correlated for simplicity. This includes Black-Scholes, Platen-Rebolledo or Vasicek models. The (self-financed) wealth process $V_{t}$ with a proportion $\alpha_{t}$ invested in stock, follows the dynamics : $d V_{t}=\alpha_{t} V_{t} \frac{d S_{t}}{S_{t}}+\left(1-\alpha_{t}\right) V_{t} \frac{d S_{t}^{0}}{S_{t}^{0}}$, and so the logarithm of the wealth process $X_{t}=\ln V_{t}$ is governed by a linear-quadratic model :

$$
d X_{t}=\left(\beta_{0} Y_{t}^{2}+\beta_{1} \alpha_{t}^{2}+\beta_{2} Y_{t} \alpha_{t}+\beta_{3} Y_{t}+\beta_{4} \alpha_{t}+\beta_{5}\right) d t+\left(\delta_{0} Y_{t}+\delta_{1} \alpha_{t}+\delta_{2}\right) d W(5
$$

where in our context, $\beta_{0}=0, \beta_{1}=-\sigma^{2} / 2, \beta_{2}=b-b_{0}, \beta_{3}=b_{0}, \beta_{4}=a-a_{0}, \beta_{5}=a_{0}, \delta_{0}=$ $0, \delta_{1}=\sigma$ and $\delta_{2}=0$. Without loss of generality, we may assume that $\sigma=1$ and so $\beta_{1}=$ $-1 / 2$ (embedded into $\alpha$ ) and $\beta_{5}=0$ (embedded into $x$ ). The P.D.E. (5.46) simplifies into the search of a pair $\left(\Lambda(\theta), \phi_{\theta}\right)$ with $\phi_{\theta}$ depending only on $y$ and solution to :

$$
\begin{aligned}
\Lambda(\theta)= & \frac{1}{2} \phi_{\theta}^{\prime \prime}-k y \phi_{\theta}^{\prime}+\frac{1}{2}\left|\phi_{\theta}^{\prime}\right|^{2}+\theta\left(\beta_{0}+\theta \frac{\delta_{0}^{2}}{2}\right) y^{2}+\theta\left(\beta_{3}+\theta \delta_{0} \delta_{2}\right) y+\theta^{2} \frac{\delta_{2}^{2}}{2} \\
& +\frac{1}{2} \frac{\theta}{1-\theta \delta_{1}^{2}}\left[\left(\beta_{2}+\theta \delta_{0} \delta_{1}\right) y+\beta_{4}+\theta \delta_{1} \delta_{2}\right]^{2} .
\end{aligned}
$$

Moreover, the maximum in $a \in \mathbb{R}$ of (5.46) is attained for

$$
\hat{\alpha}(\theta, y)=\frac{\left(\beta_{2}+\theta \delta_{0} \delta_{1}\right) y+\beta_{4}+\theta \delta_{1} \delta_{2}}{1-\theta \delta_{1}^{2}} .
$$


The above calculations are valid only for $0 \leq \theta<1 / \delta_{1}^{2}$. We are looking for a quadratic solution to the ordinary differential equation (5.48) :

$$
\phi_{\theta}(y)=\frac{1}{2} A(\theta) y^{2}+B(\theta) y \text {. }
$$

By substituting into (5.48), and cancelling terms in $y^{2}, y$ and constant terms, we obtain

- a polynomial second degree equation for $A(\theta)$

- a linear equation for $B(\theta)$, given $A(\theta)$

- $\Lambda(\theta)$ is then expressed explicitly in function of $A(\theta)$ and $B(\theta)$ from (5.48).

The existence of a solution to the second degree equation for $A(\theta)$, through the nonnegativity of the discriminant, allows to determine the bound $\bar{\theta}$ and so the interval $[0, \bar{\theta})$ on which $\Lambda$ is well-defined and finite. Moreover, we find two possible roots to the polynomial second degree equation for $A(\theta)$, but only one satisfies the ergodicity condition. From Theorem 5.2, we deduce that

$$
v(x)=-\sup _{\theta \in[0, \bar{\theta})}[\theta x-\Lambda(\theta)], \quad \forall x<\Lambda^{\prime}(\bar{\theta}),
$$

with a sequence of nearly optimal controls given by :

$$
\alpha_{t}^{*, n}=\left\{\begin{aligned}
\hat{\alpha}\left(\theta\left(x+\frac{1}{n}\right), Y_{t}\right), & \Lambda^{\prime}(0) & <x<\Lambda^{\prime}(\bar{\theta}) \\
\hat{\alpha}\left(\theta\left(\Lambda^{\prime}(0)+\frac{1}{n}\right), Y_{t}\right), & x & \leq \Lambda^{\prime}(0),
\end{aligned}\right.
$$

with $\theta(x) \in(0, \bar{\theta})$ s.t. $\Lambda^{\prime}(\theta(x))=x$. In the one-factor model described above, the function $\Lambda$ is steep, i.e. $\Lambda^{\prime}(\bar{\theta})=\infty$, and so (5.50) holds for all $x \in \mathbb{R}$. For example, in the Black-Scholes model, i.e. $b_{0}=b=0$, we obtain

$$
\begin{aligned}
& \Gamma(\theta)=\Lambda(\theta)=\frac{1}{2} \frac{\theta}{1-\theta}\left(\frac{a-a_{0}}{\sigma^{2}}\right)^{2}, \quad \text { for } \theta<\bar{\theta}=1, \\
& v(x)=-\sup _{\theta \in[0,1)}[\theta x-\Gamma(\theta)]=\left\{\begin{array}{cl}
-(\sqrt{x}-\sqrt{\bar{x}})^{2}, & \text { if } x \geq \bar{x}:=\Gamma^{\prime}(0)=\frac{1}{2}\left(\frac{a-a_{0}}{\sigma^{2}}\right)^{2} \\
0, & \text { if } x<\bar{x},
\end{array}\right.
\end{aligned}
$$

$\theta(x)=1-\sqrt{\bar{x} / x}$ if $x \geq \bar{x}$, and 0 otherwise, and

$$
\alpha_{t}^{*}= \begin{cases}\sqrt{2 x}, & \text { if } x \geq \bar{x} \\ \frac{a-a_{0}}{\sigma^{2}}, & \text { if } x<\bar{x}\end{cases}
$$

We observe that for an index value $x$ small enough, actually $x<\bar{x}$, the optimal investment for our large deviations criterion is equal to the optimal investment of the Merton's problem for an investor with relative risk aversion one. When the value index is larger than $\bar{x}$, the optimal investment is increasing with $x$, with a degree of relative risk aversion $1-\theta(x)$ decreasing in $x$.

In the more general linear-quadratic model (5.47), $\Lambda$ may be steep or not depending on the parameters $\beta_{i}$ and $\delta_{i}$. We refer to [37] for the details. Some variants and extensions of this large deviations control problem are studied in [30] and [1]. 


\section{Conclusion}

In these notes, we developed some applications and emphasized methods of large deviations in finance and insurance. These applications are multiple, and our presentation is by no means exhaustive. There are numerous works dealing with large deviations techniques in the context of insurance, see e.g. [14], 四], or more recently [33] and [35]. We also cite the paper [6], which develops asymptotic formula for calculating implied volatility of index options. Large deviation principle for backward stochastic differential equations is used by [5] in a setting motivated by credit risk management. Other papers using large deviations in portfolio management are [38] and [8]. Some aspects of large deviations applied to problems in macroeconomics are studied in [41].

From a general viewpoint, questions related to extremal events are embedded into the extreme value theory, and we refer to the classical book [16] for a development of this subject, especially regarding applications in finance and insurance.

\section{References}

[1] Akian M., Gaubert S. and V. Kolokoltsov (2005) : "Solutions of max-plus linear equations and large deviations", Proceedings of the joint 44th IEEE Conference on Decision and Control and European Control Conference ECC 2005.

[2] Arouna B. (2004) : "Adaptative Monte-Carlo methods, a variance reduction technique", Montecarlo Methods and Applications, 10, 1-24.

[3] Asmussen S. (2000) : Ruin Probabilities, World Scientific.

[4] Asmussen S. and C. Kluppelberg (1996) : "Large deviation results for subexponential tails, with applications to insurance risk", Stoch. Proc. Appl., 64, 103-125.

[5] Astic F. and S. Rainero (2006) : "Conditional large deviations and applications to credit risk management", Preprint University Paris Dauphine.

[6] Avellaneda M., Boyer-Olson D., Busca J. and P. Friz (2003) : "Méthodes de grandes déviations et pricing d'options sur indice", C.R. Acad. Sci. Paris, 336, 263-266.

[7] Baldi P., Caramellino L. and M. Iovino (1999) : "Pricing general barrier options: a numerical approach using sharp large deviations", Mathematical Finance, 9, 293-322.

[8] Bares P., Cont R., Gardiol L., Gibson R. and S. Gyger (2000) : "A large deviation approach to portfolio management", International Journal of Theoretical and Applied Finance, 3, 617-639.

[9] Barles G. (1994) : Solutions de viscosité des équations d'Hamilton-Jacobi, Springer Verlag.

[10] Bielecki T. and S. Piska (2004) : "Risk-sensitive ICAPM with application to fixed-income management", IEEE Transactions on automatic control, 49, 420-432.

[11] Browne S. (1999) : "Beating a moving target : optimal portfolio strategies for outperforming a stochastic benchmark", Finance and Stochastics, 3, 275-294.

[12] Dembo A., Deuschel J.D. and D. Duffie (2004), "Large portfolio losses", Finance and Stochastics, 8, 3-16. 
[13] Dembo A. and O. Zeitouni (1998) : Large deviations techniques and applications, 2nd edition, Springer Verlag.

[14] Djehiche B. (1993) : "A large deviation estimate for ruin probabilities", Scandinavian Actuarial Journal, 1, 42-59.

[15] Dupuis P. and R. Ellis (1997) : A weak convergence approach to the theory of large deviations, Wiley Series in Probability and Statistics.

[16] Embrechts P., Kluppelberg C. and T. Mikosch (2003), Modelling extremal events for insurance and finance, 4th edition, Springer Verlag.

[17] Fleming W. and M. James (1992) : "Asymptotic series and exit time probability", Annals of Probability, 20, 1369-1384.

[18] Fleming W. and W. McEneaney (1995) : "Risk sensitive control on an infinite time horizon", SIAM Journal on Control and Optimization, 33, 1881-1915.

[19] Fleming W. and M. Soner (1994) : Controlled Markov processes and viscosity solutions, Springer Verlag.

[20] Föllmer H. and P. Leukert (1999) : "Quantile hedging", Finance and Stochastics, 3, 251-273.

[21] Fournié E., Lasry J.M. and P.L. Lions (1997) : "Some nonlinear methods to study far-fromthe-money contingent claims", Numerical Methods in Finance, L.C.G. Rogers et D. Talay, eds, Cambridge University Press.

[22] Fournié E., Lasry J.M. and N. Touzi (1997) : "Monte Carlo methods for stochastic volatility models", Numerical Methods in Finance, L.C.G. Rogers et D. Talay, eds, Cambridge University Press.

[23] Gaier J., Grandits P. and W. Schachermayer (2003) : "Asymptotic ruin probabilities and optimal investment", Annals of Applied Probability, 13, 1054-1076.

[24] Gerber H. (1973) : "Martingales in risk theory", Mitt. Ver. Schweiz. math., 73, 205-216.

[25] Glasserman P., Heidelberger P. and P. Shahabuddin (1999), "Asymptotically optimal importance sampling and stratification for pricing path-dependent options", Mathematical finance, $\mathbf{9}$, $117-152$.

[26] Glasserman P., Kang W. and P. Shahabuddin (2006), "Large deviations in multifactor portfolio credit risk", to appear in Mathematical Finance.

[27] Glasserman P. and J. Li (2005), "Importance sampling for portfolio credit risk", Management science, 51, 1643-1656.

[28] Gobet E. (2000) : "Weak approximations of killed diffusion using Euler schemes", Stochastic Processes and their Applications, 87, 167-197.

[29] Guasoni P. and S. Robertson (2006) : "Optimal importance sampling with explicit formulas in continuous-time", Preprint, Boston University.

[30] Hata H. and J. Sekine (2005) : "Solving long term invesmtment problems with Cox-IngersollRoss interest rates", Advances in Mathematical Economics, 8, 231-255.

[31] Hipp C. and H. Schmidli (2004) : "Asymptotics of ruin probabilities for controlled risk processes in the small claims case", Scandinavian Actuarial Journal, 321-335.

[32] Huh J. and A. Kolkiewicz (2006) : "Efficient computation of multivariate barrier crossing probability and its applications in credit risk models", Preprint University of Waterloo. 
[33] Kaas R. and Q. Tang (2005) : "A large deviation result for aggregate claims with dependent claims occurences", Insurance Mathematics and Economics, 36, 251-259.

[34] Lasry J.M. and P.L. Lions (1995) : "Grandes déviations pour des processus de diffusion couplés par un processus de sauts", CRAS, t. 321, 849-854.

[35] Macci C. and G. Stabile (2006) : "Large deviations for risk processes with reinsurance", Journal of Applied Probability, 43, 713-728.

[36] Pham H. (2003a) : "A large deviations approach to optimal long term investment", Finance and Stochastics, 7, 169-195.

[37] Pham H. (2003b) : "A risk-sensitive control dual approach to a large deviations control problem", Systems and Control Letters, 49, 295-309.

[38] Sornette D. (1998) : "Large deviations and portfolio optimization", Physica A : Statistical and Theoretical Physics, 256, 251-283.

[39] Stettner L. (2004) : "Duality and risk sensitive portfolio optimization", in Mathematics of Finance, Proceedings AMS-IMS-SIAM, eds G. Yin and Q. Zhang, 333-347.

[40] Stutzer M. (2003) : "Portfolio choice with endogenous utility : a large deviations approach", Journal of Econometrics, 116, 365-386.

[41] Williams N. (2004) : "Small Noise Asymptotics for a Stochastic Growth Model", Journal of Economic Theory, 119, 271-298. 